\title{
Perturbative degrees of freedom in loop quantum gravity: anisotropies
}

\author{
Martin Bojowald $^{1,3}$, Héctor H Hernández ${ }^{1}$ and Hugo A Morales Técott ${ }^{2,4}$ \\ ${ }^{1}$ Max-Planck-Institut für Gravitationsphysik, Albert-Einstein-Institut, Am Mühlenberg 1, \\ D-14476 Potsdam, Germany \\ 2 Departamento de Física, Universidad Autónoma Metropolitana Iztapalapa, AP 55-534, \\ México DF 09340, Mexico \\ E-mail: bojowald@gravity.psu.edu,hehe@aei.mpg.de and hugo@xanum.uam.mx
}

Received 16 November 2005

Published 28 April 2006

Online at stacks.iop.org/CQG/23/3491

\begin{abstract}
The relation between an isotropic model and an anisotropic model in loop quantum cosmology is discussed in detail, comparing the strict symmetry reduction with a perturbative implementation of symmetry. While the latter cannot be done in a canonical manner, it allows one to consider the dynamics including the role of small non-symmetric degrees of freedom for the symmetric evolution. This serves as a model for the general situation of perturbative degrees of freedom in a background-independent quantization such as loop quantum gravity, and for the more complicated addition of perturbative inhomogeneities. While being crucial for cosmological phenomenology, it is shown that perturbative non-symmetric degrees of freedom do not allow definitive conclusions for the singularity issue and in such a situation could even lead to wrong claims.
\end{abstract}

PACS numbers: $04.60 . \mathrm{Pp}, 04.60 . \mathrm{Kz}$

\section{Introduction}

By far the most common and extended studies in cosmological models are those admitting only finitely many or even one gravitational degree of freedom, the scale factor or radius of the universe $a(t)$ and maybe anisotropy parameters. This is justified in a homogeneous or isotropic model, symmetries present in our universe at large scales. This is also mainly implemented in models of quantum gravity, such as in Wheeler-DeWitt quantum cosmology $[1,2]$ and loop quantum cosmology [3-5]. That degree of symmetry does not only allow

\footnotetext{
3 Present address: Institute for Gravitational Physics and Geometry, 104 Davey Lab., The Pennsylvania State University, University Park, PA 16802, USA.

4 Associated member of ICTP, Trieste, Italy. 
insights into phenomenological aspects of the early universe but, in anisotropic models, also displays the most crucial features of classical singularities.

More realistic models can then be obtained by breaking some of the symmetries imposed as before. After isotropy the first step is anisotropy, which can often be implemented exactly, and then inhomogeneity as the crucial one. In the first case (anisotropy), there is still a finite number of degrees of freedom and usually they do not pose much new technical difficulty. Conceptually, however, they are essential in particular for a general understanding of the issue of singularities (see, e.g., [6] for a recent discussion in the context of quantum cosmology). It is then of interest to discuss anisotropic models at different levels and to see what the implications for singularities are. Complete quantizations have been done in [7-9] with the result that their loop quantizations are free of singularities as in isotropic cases [10-12].

Alternatively, one can try to implement anisotropies as perturbations to a quantized isotropic model and see how correction terms change the dynamical behaviour. In this work, we develop such a point of view both to shed light on the singularity issue and as a model for perturbative inhomogeneities. Following [7] where anisotropic models have been quantized in the loop framework, we introduce suitable anisotropy parameters in that model and implement conditions for them to be small. (As a first step we take only one anisotropy parameter of two possible ones which means that there is still a single rotational symmetry axis, a so-called locally rotationally symmetric (LRS) situation.) This will be discussed in detail at the kinematical and dynamical levels and results in an evolution equation describing an anisotropic model perturbatively around an isotropic one. The formulation can also be used for comparing background-independent theories with theories formulated on a background, because the isotropic model can be seen as a background for the perturbative anisotropy.

In section 2, we describe the classical models, to be quantized in section 3 both exactly and in a perturbative manner. This illustrates how symmetric models can be related to a less symmetric theory at the quantum level. In section 4 , we discuss the evolution equation in terms of the perturbation and its new features compared to the isotropic model. In section 5, we look at the singularity issue from the perturbative point of view and show that conclusions drawn in such a situation must be used much more carefully than in a non-perturbative quantization.

\section{Classical models}

For simplicity, we consider the two simplest models, which are the flat isotropic model and the Bianchi I model with one additional rotational symmetry (LRS). Since loop quantizations are based on Ashtekar variables $[13,14]$ given by a connection $A_{a}^{i}$ and a densitized triad $E_{i}^{a}$, we first introduce these variables. In models, they take special forms corresponding to the fact that they are required to be preserved by a symmetry transformation up to a gauge transformation.

An isotropic connection and densitized triad for a flat model can always be written as

$$
\begin{aligned}
& A_{a}{ }^{i} \mathrm{~d} x^{a}=\bar{c} \mathrm{~d} x^{i} \\
& E_{i}{ }^{a} \frac{\partial}{\partial x^{a}}=\bar{p} \frac{\partial}{\partial x^{i}},
\end{aligned}
$$

in Cartesian coordinates, where the only two remaining components are conjugate to each other,

$$
\{\bar{c}, \bar{p}\}=\frac{8 \pi}{3} \gamma G
$$

with the Barbero-Immirzi parameter $\gamma[14,15]$ and the gravitational constant $G$. The variable $\bar{p}$ contains information about spatial geometry such as the volume

$$
V_{\text {iso }}=|\bar{p}|^{3 / 2}
$$


while $\bar{c}$ is proportional to extrinsic curvature. Both variables are related dynamically as dictated by the Hamiltonian constraint

$$
H_{\text {iso }}=-\frac{3}{8 \pi G \gamma^{2}} \bar{c}^{2} \sqrt{|\bar{p}|}+H_{\text {matter }}(\bar{p})=0 .
$$

Similarly, in the LRS Bianchi I model we have

$$
\begin{aligned}
& A_{a}^{i} \mathrm{~d} x^{a} \tau_{i}=A \tau^{1} \mathrm{~d} x+A \tau^{2} \mathrm{~d} y+c \tau^{3} \mathrm{~d} z \\
& E_{i}^{a} \frac{\partial}{\partial x^{a}} \tau^{i}=p_{A} \tau^{1} \partial_{x}+p_{A} \tau^{2} \partial_{y}+p_{c} \tau^{3} \partial_{z}
\end{aligned}
$$

with a symplectic structure

$$
\left\{A, p_{A}\right\}=4 \pi \gamma G, \quad\left\{c, p_{c}\right\}=8 \pi \gamma G .
$$

There is a residual gauge transformation $p_{A} \mapsto-p_{A}$ which can be fixed by requiring $p_{A} \geqslant 0$ (see $[7,9]$ for more details). The volume is now given by

$$
V_{\text {aniso }}=\sqrt{p_{A}^{2}\left|p_{c}\right|}
$$

and the Hamiltonian constraint by

$$
H_{\text {aniso }}=-(8 \pi G)^{-1} \gamma^{-2}\left(A^{2} \sqrt{p_{A}}+2 A c \sqrt{\left|p_{c}\right|}\right)+H_{\text {matter }}\left(p_{A}, p_{c}\right)=0 .
$$

To identify the anisotropy as a perturbation it is convenient to introduce new variables

$$
\begin{aligned}
& (A, c)=(\bar{c}+\varepsilon, \bar{c}-2 \varepsilon) \\
& \left(p_{A}, p_{c}\right)=\left(\bar{p}+p_{\varepsilon}, \bar{p}-2 p_{\varepsilon}\right)
\end{aligned}
$$

together with the inverse transformation

$$
\begin{aligned}
& (\bar{c}, \bar{p})=\left(\frac{1}{3}(2 A+c), \frac{1}{3}\left(2 p_{A}+p_{C}\right)\right) \\
& \left(\varepsilon, p_{\varepsilon}\right)=\left(\frac{1}{3}(A-c), \frac{1}{3}\left(p_{A}-p_{C}\right)\right)
\end{aligned}
$$

as well as the symplectic structure

$$
\{\bar{c}, \bar{p}\}=\frac{8 \pi}{3} \gamma G, \quad\left\{\varepsilon, p_{\varepsilon}\right\}=\frac{4 \pi}{3} \gamma G .
$$

The volume, in this manner, is given by $\bar{p}$ up to terms of at least second order in $p_{\varepsilon}$, which is the motivation for choosing our particular set of anisotropy variables:

$$
V_{\text {aniso }}=\sqrt{\left|\bar{p}^{3}-3 p_{\varepsilon}^{2} \bar{p}-2 p_{\varepsilon}^{3}\right|}=|\bar{p}|^{3 / 2}\left(1-\frac{3}{2} \frac{p_{\varepsilon}^{2}}{\bar{p}^{2}}+O\left(p_{\varepsilon}^{3} / \bar{p}^{3}\right)\right) .
$$

This shows that we have to assume $p_{\varepsilon} \ll \bar{p}$ for perturbations, i.e. the approximation will break down close to classical singularities of the isotropic type where $\bar{p}=0$. Later, we will also have to assume $\varepsilon \ll 1$. For the Hamiltonian constraint we obtain

$H_{\text {aniso }}=-\frac{3}{8 \pi G \gamma^{2}}|\bar{p}|^{-3 / 2}\left(\bar{c}^{2} \bar{p}^{2}-\varepsilon^{2} \bar{p}^{2}-\bar{c}^{2} p_{\varepsilon}^{2}+2 \bar{c} \bar{p} \varepsilon p_{\varepsilon}+O\left(p_{\varepsilon}^{3} / \bar{p}^{3}\right)\right)+H_{\text {matter }}\left(\bar{p}, p_{\varepsilon}\right)$

up to terms of third order in the perturbation. 
In the vacuum case, we have Hamiltonian equations of motion

$$
\begin{aligned}
& \dot{\bar{c}}=\left\{\bar{c}, H_{\text {aniso }}\right\} \propto-\bar{c}^{2} \bar{p}+\varepsilon^{2} \bar{p}-\bar{c} \varepsilon p_{\varepsilon} \\
& \dot{\bar{p}}=\left\{\bar{p}, H_{\text {aniso }}\right\} \propto \bar{c} \bar{p}^{2}-\bar{c} p_{\varepsilon}^{2}+\bar{p} \varepsilon p_{\varepsilon} \\
& \dot{\varepsilon}=\left\{\varepsilon, H_{\text {aniso }}\right\} \propto \frac{1}{2} \bar{c}^{2} p_{\varepsilon}-\frac{1}{2} \bar{c} \bar{p} \varepsilon \\
& \dot{p}_{\varepsilon}=\left\{p_{\varepsilon}, H_{\text {aniso }}\right\} \propto-\frac{1}{2} \bar{p}^{2} \varepsilon+\frac{1}{2} \bar{c} \bar{p} p_{\varepsilon} .
\end{aligned}
$$

Thus, the isotropic variables receive corrections in their equations of motion only at second order. Ignoring those corrections, we recover the classical solution $\bar{c}=0$ and $\bar{p}=$ const for flat Minkowski space. With this approximate solution for the anisotropic system, the equations for anisotropies simplify to $\dot{\varepsilon} \approx 0$ and $\dot{p}_{\varepsilon} \approx-\frac{1}{2} \bar{p}^{2} \varepsilon$. The anisotropy in the connection will thus remain constant while that in the triad grows linearly in time until the approximations break down. Since the constraint is quadratic in connection components, a feature shared by the full constraint, we are not required at this point to assume $\varepsilon \ll 1$. However, the equations of motion show that $\dot{p}_{\varepsilon}$ would be large if $\varepsilon$ is not small, and we are led to this condition if the perturbative evolution is to be valid for some time.

We will later, at the quantum level, mainly consider an asymptotic approximation of very large $\bar{p}$ where all corrections of higher order in $p_{\varepsilon} / \bar{p}$ are ignored. This also simplifies the classical equations because the Hamiltonian constraint is then simply proportional to $-\left(\bar{c}^{2}-\varepsilon^{2}\right) \sqrt{|\bar{p}|}$. Note that the quadratic nature of the constraint in connection variables makes this approximation reasonable as we do not need to expand in $\varepsilon$. The solution $\varepsilon=\bar{c}$ to the constraint is thus consistent even though the anisotropy $\varepsilon$ in the connection is not small compared to $\bar{c}$ (unlike $p_{\varepsilon}$ which must be small compared to $\bar{p}$ ). As already mentioned, and used later, it is only necessary that $\varepsilon$ is small compared to one, which is usually the case also for $\bar{c}$ in semiclassical regimes.

Using the large- $\bar{p}$ constraint we have equations of motion

$$
\begin{aligned}
& \dot{\bar{c}}=-\frac{\bar{c}^{2}}{2 \sqrt{|\bar{p}|}}+\frac{\varepsilon^{2}}{2 \sqrt{|\bar{p}|}} \approx 0 \\
& \dot{\bar{p}}=2 \bar{c} \sqrt{|\bar{p}|} \\
& \dot{\varepsilon}=0 \\
& \dot{p}_{\varepsilon}=-\varepsilon \sqrt{|\bar{p}|} .
\end{aligned}
$$

With $\varepsilon=\bar{c}=$ const we have $\bar{p}=\left(\varepsilon t+\sqrt{\left|\bar{p}_{0}\right|}\right)^{2}$ and $p_{\varepsilon}=p_{\varepsilon}^{0}-\frac{1}{2} \varepsilon^{2} t^{2}-\varepsilon \sqrt{\left|\bar{p}_{0}\right|} t$ with constants of integration $\bar{p}_{0}$ and $p_{\varepsilon}^{0}$. Eliminating $t$, we have the internal time evolution

$$
p_{\varepsilon}(\bar{p})=p_{\varepsilon}^{0}-\frac{1}{2}\left(\bar{p}-\bar{p}_{0}\right)
$$

of $p_{\varepsilon}$ with respect to $\bar{p}$. Note that this evolution, unlike the coordinate time evolution, does not depend on the value of $\varepsilon$. We will use this fact later as a test of the perturbative quantum evolution.

\section{Quantization}

Loop quantizations are based on holonomies, and also in models one uses exponentials of connection components as basic expressions in addition to densitized triad components. In the isotropic case, this leads to a basic algebra given by $p$ and $\exp (\mathrm{i} \mu \bar{c} / 2)$ for all $\mu \in \mathbb{R}$, represented on a non-separable Hilbert space

$$
\mathcal{H}_{\text {iso }} \cong L^{2}\left(\overline{\mathbb{R}}_{\text {Bohr }}, \mathrm{d} \mu_{\mathrm{H}}\right)
$$


of square-integrable functions on the Bohr compactification of the real line [12], with orthonormal basis

$$
\langle\bar{c} \mid \mu\rangle=\exp (\mathrm{i} \mu \bar{c} / 2), \quad \mu \in \mathbb{R}
$$

and basic operators acting as

$$
\begin{aligned}
& \left.\operatorname{exp(\mathrm {i}\mu ^{\prime }\overline {c}} / 2\right)|\mu\rangle=\left|\mu+\mu^{\prime}\right\rangle \\
& \hat{\bar{p}}|\mu\rangle=\frac{1}{6} \gamma \ell_{\mathrm{P}}^{2} \mu|\mu\rangle
\end{aligned}
$$

using the Planck length $\ell_{\mathrm{P}}=\sqrt{8 \pi G \hbar}$. Similarly, for our anisotropic model we have the Hilbert space

$$
\mathcal{H}_{\text {aniso }} \cong \mathcal{H}_{\text {iso }} \otimes \mathcal{H}_{\text {iso }}
$$

with orthonormal basis

$$
\langle A, c \mid \mu, v\rangle=\exp (\mathrm{i}(\mu c+\nu A) / 2), \quad \mu, \nu \in \mathbb{R}
$$

holonomy operators as before and

$$
\hat{p}_{A}|\mu, v\rangle=\frac{1}{4} \gamma \ell_{\mathrm{P}}^{2} \mu|\mu, v\rangle, \quad \hat{p}_{c}|\mu, v\rangle=\frac{1}{2} \gamma \ell_{\mathrm{P}}^{2} \nu|\mu, v\rangle .
$$

These states are not completely gauge invariant in view of the residual gauge transformation $\mu \mapsto-\mu$, which however will not be relevant for our purposes. One can always ensure gauge invariance by working only with states $\sum_{\mu, \nu} \psi_{\mu, \nu}|\mu, \nu\rangle$, where $\psi_{\mu, \nu}$ is symmetric in $\mu$. The triad operators then give us directly the volume operators for both models by inserting them into the classical expressions.

For mathematical constructions, one often makes use of dense subspaces $\mathrm{Cyl}_{\text {iso }}$ and $\mathrm{Cyl}_{\text {aniso }}$ of the two Hilbert spaces given by the so-called cylindrical functions which are finite linear combinations of the basis states $|\mu\rangle$ or $|\mu, \nu\rangle$, respectively. With their algebraic duals of distributional states (linear functionals on the cylindrical subspaces), one has two Gel'fand triples $\mathrm{Cyl}_{\text {iso }} \subset \mathcal{H}_{\text {iso }} \subset \mathrm{Cyl}_{\text {iso }}^{\star}$ and $\mathrm{Cyl}_{\text {aniso }} \subset \mathcal{H}_{\text {aniso }} \subset \mathrm{Cyl}_{\text {aniso }}^{\star}$ (see also [16]).

\subsection{Symmetry reduction}

Since we are interested in the relation between a symmetric model and a less symmetric one we first use the opportunity to demonstrate how a reduced model can be obtained within quantum theory (see also $[5,17,18]$ ). We start with the anisotropic model and, following general constructions [17], define an isotropic state as a distribution which is supported only on isotropic connections, i.e. $\varepsilon=0$. To fulfil the definition, we must find an antilinear map

$$
\sigma: \quad \mathrm{Cyl}_{\text {iso }} \rightarrow \mathrm{Cyl}_{\text {aniso }}^{\star}, \quad|\mu\rangle \mapsto(\mu \mid
$$

embedding the space of isotropic cylindrical functions into the distributional dual of the anisotropic one such that

$$
\sigma(|\mu\rangle)[|\rho, \tau\rangle]=\left.\langle\mu \mid \rho, \tau\rangle\right|_{A=c} \quad \text { for all }|\rho, \tau\rangle .
$$

On the right-hand side, we use the restriction of a state $|\rho, \tau\rangle$ to the subspace $A=c$ upon which it can be interpreted as an isotropic state. Expanding $\sigma(|\mu\rangle)=: \sum_{\kappa, \lambda} \sigma_{\kappa, \lambda}(\mu)\langle\kappa, \lambda|$, we have

$$
\sigma_{\rho, \tau}(\mu)=\sum_{\kappa, \lambda} \sigma_{\kappa, \lambda}(\mu) \delta_{\kappa, \rho} \delta_{\lambda, \tau}=\sigma(|\mu\rangle)[|\rho, \tau\rangle]=\int \mathrm{e}^{-\mathrm{i} \mu c / 2} \mathrm{e}^{\mathrm{i}(\rho+\tau) c / 2} \mathrm{~d} \mu_{\mathrm{H}}(c)=\delta_{\mu, \rho+\tau}
$$


and thus

$$
\left(\mu \mid=\sigma(|\mu\rangle)=\sum_{\kappa, \lambda} \delta_{\mu, \kappa+\lambda}\langle\kappa, \lambda|=\sum_{\kappa}\langle\kappa, \mu-\kappa| .\right.
$$

The summation is done over all real numbers, which is well defined as a distribution in $\mathrm{Cyl}_{\text {aniso }}^{\star}$.

In fact, in this case the definition simply amounts to multiplying an isotropic state in the connection representation with a $\delta$ distribution supported at $\varepsilon=0$,

$$
\sigma(|\mu\rangle)|A, c\rangle=\sum_{\kappa} \mathrm{e}^{\mathrm{i}(\kappa A+(\mu-\kappa) c) / 2}=\mathrm{e}^{\mathrm{i} \mu c / 2} \sum_{\kappa} \mathrm{e}^{\mathrm{i} \kappa(A-c) / 2}=\mathrm{e}^{\mathrm{i} \mu c / 2} \delta(A-c)
$$

but this would be more complicated in more general systems and the full theory where the definitions of [17] still apply.

Indeed, this gives $\left(\mu|\rho, \tau\rangle=\delta_{\mu, \rho+\tau}\right.$, i.e. the evaluation of the distribution is nonzero only if the averaged label equals the isotropic one,

$$
\frac{1}{2} \gamma \ell_{\mathrm{P}}^{2}(\rho+\tau)=2\left(\hat{p}_{A}\right)_{\rho}+\left(\hat{p}_{c}\right)_{\tau}=3(\hat{\bar{p}})_{\rho+\tau}
$$

and $\left(\mu \mid h_{A}^{(\rho)} h_{c}^{(\rho)-1}=(\mu \mid\right.$, i.e. $\exp (\mathrm{i} \rho \varepsilon)$ acts trivially with its dual action on symmetric distributions which are thus supported only on isotropic connections $\varepsilon=0$.

Here, we already used the dual action of anisotropic operators on isotropic distributional states, which in this example mapped a symmetric state to a symmetric one. This is not the case for arbitrary operators, and one cannot simply define all operators for the reduced model by the dual action of anisotropic operators [18]. However, one can do this for special operators which suffice to define the basic operators of the model. For flux operators, one can easily see that $2 \hat{p}_{A}+\hat{p}_{c}=3 \hat{\bar{p}}$ is the only one which maps an isotropic state $(\mu \mid$ to an isotropic state:

$$
\hat{p} \sigma(|\mu\rangle)=\frac{1}{3}\left(\mu \mid\left(2 \hat{p}_{A}+\hat{p}_{c}\right)=\frac{1}{6} \gamma \ell_{\mathrm{P}}^{2} \mu(\mu \mid=\sigma(\hat{\bar{p}}|\mu\rangle) .\right.
$$

Moreover, it agrees with the isotropic flux operator (26) defined in the isotropic model. For holonomies, one can act with arbitrary products of $h_{A}$ and $h_{c}$ on isotropic states since we already know that $h_{A} h_{c}^{-1}$ acts as the identity and the remaining factor simply amounts to an isotropic holonomy operator. Also, all holonomy operators form a closed algebra with the isotropic flux operator $\hat{\bar{p}}=\frac{1}{3}\left(2 \hat{p}_{A}+\hat{p}_{c}\right)$ :

$\left[h_{A}^{(\rho)} h_{c}^{(\tau)}, \hat{\bar{p}}\right]|\mu, \nu\rangle=-\frac{1}{6} \gamma \ell_{\mathrm{P}}^{2}(\rho+\tau)|\mu+\rho, \nu+\tau\rangle=-\frac{1}{6} \gamma \ell_{\mathrm{P}}^{2}(\rho+\tau) h_{A}^{(\rho)} h_{c}^{(\tau)}|\mu, \nu\rangle$.

This is similar for the anisotropy operator which we can define by $\hat{p}_{\varepsilon}:=\frac{1}{3}\left(\hat{p}_{A}-\hat{p}_{c}\right)$. Here, we have the commutator

$$
\left[h_{A}^{(\rho)} h_{c}^{(\tau)}, \hat{p}_{\varepsilon}\right]=-\frac{1}{6} \gamma \ell_{\mathrm{P}}^{2}\left(\frac{1}{2} \rho-\tau\right) h_{A}^{(\rho)} h_{c}^{(\tau)} .
$$

However, $\hat{p}_{\varepsilon}$ does not map an isotropic distribution to another such distribution.

If we are looking for a reduction of the operator algebra to implement isotropy, we need to find distinguished dual actions making use of the form of symmetric states. One clearly distinguished operator is $\hat{\bar{p}}$ because it is the only flux operator mapping an isotropic distributional state to another such state. For holonomies, $h_{A} h_{c}^{-1}$ is distinguished because it fixes any isotropic distributional state. Beyond that, no other operators are intrinsically distinguished, but we do not yet have a useful algebra of operators for an isotropic model: while $\bar{p}$ is available, our distinguished holonomy corresponds to the anisotropy $\varepsilon$ rather than the conjugate of $\bar{p}$. Nevertheless, by acting with the distinguished $\hat{p}$ as well as all holonomy operators, we can generate the isotropic representation. More precisely, we define

$$
\operatorname{Stab}(\sigma):=\left\{\hat{O} \in \mathcal{A}_{\text {aniso }} \mid \hat{O} \sigma\left(\mathrm{Cyl}_{\text {iso }}\right) \subset \sigma\left(\mathrm{Cyl}_{\text {iso }}\right)\right\}
$$

and

$$
\operatorname{Fix}(\sigma):=\left\{\hat{O} \in \mathcal{A}_{\text {aniso }} \mid \hat{O} \sigma(\psi)=\sigma(\psi) \text { for all } \psi \in \mathrm{Cyl}_{\text {iso }}\right\}
$$


The isotropic algebra is then

$$
\mathcal{A}_{\text {iso }}:=\operatorname{Stab}(\sigma) / \operatorname{Fix}(\sigma)
$$

understood in the following manner: $\operatorname{Fix}(\sigma)$ contains only holonomy operators besides the identity, and thus forms a normal subgroup of the Abelian group obtained from the multiplicative operation in the holonomy algebra. We can thus take the factor group of holonomy operators modulo Fix $(\sigma)$ which thanks to the distributive law can also be equipped with the usual additive structure to form an algebra. Due to the relation (33), also the flux operators in $\operatorname{Stab}(\sigma)$ commute with $\operatorname{Fix}(\sigma)$ and thus descend to the factor group, defining $\mathcal{A}_{\text {iso }}$. Using that $\operatorname{Stab}(\sigma)$ is generated as an algebra by $\hat{\bar{p}}$ and all $h_{A}^{(\rho)} h_{c}^{(\tau)}$ and Fix $(\sigma)$ by $h_{A} h_{c}^{-1}$, a general element of $\mathcal{A}_{\text {iso }}$ is

$$
\left(\sum_{n \in \mathbb{N}_{0}} \hat{\bar{p}}^{n} \pi_{n}\left(h_{c}\right)\right) \cdot \operatorname{Fix}(\sigma)
$$

with Laurent polynomials $\pi_{n}$, allowing also inverse powers of $h_{c}$, for each $n$, which are nonzero only for finitely many $n$ (we can order $\hat{p}$ to the left using (33) and factor out a power of $h_{A} h_{c}^{-1}$ into Fix $(\sigma)$ such that only the holonomy $h_{c}$ appears explicitly). Through this construction we derive an algebra isomorphic to the isotropic one.

As the presence of factor spaces indicates, there is no canonical isotropic subalgebra of the anisotropic algebra without additional input. This is analogous to the classical situation where the splitting of anisotropic variables $A$ and $c$ into an isotropic average $\bar{c}$ and an anisotropy is not unique. Classically, a given $\bar{p}$, as we have distinguished it at the quantum level, determines a form of $\varepsilon$ as a linear combination of $A$ and $c$ by requiring $\{\bar{p}, \varepsilon\}=0$. Indeed, also the quantum analogue of $\varepsilon$, given by $h_{A} h_{c}^{-1}$, is distinguished as it commutes with $\hat{\bar{p}}$. However, a unique form for $\bar{c}$ and $p_{\varepsilon}$ then follows only after an additional choice, which for us was the fact that the volume should receive corrections only to second order. We use this here to define $\hat{p}_{\varepsilon}=\frac{1}{3}\left(\hat{p}_{A}-\hat{p}_{c}\right)$ as above and then require that (34) vanish, analogously to $\left\{\bar{c}, p_{\varepsilon}\right\}=0$. This distinguishes $h_{A}^{2} h_{c}$ as the isotropic holonomy operator in addition to the flux $\hat{\bar{p}}$ which indeed forms a subalgebra of the anisotropic operator algebra mapping isotropic states to isotropic states in a way isomorphic to the isotropic model.

In this manner, we obtain a unique subalgebra of the basic anisotropic operator algebra which is isomorphic to the basic isotropic algebra. This also presents an alternative way to define a symmetric model without referring to symmetric distributional states at all: we use the classical relations between symmetric and non-symmetric variables to define a distinguished algebra derived from the non-symmetric holonomy-flux algebra. Using the fact that the nonsymmetric representation is cyclic on $|0,0\rangle$, i.e. the subspace $\mathcal{A}_{\text {aniso }}|0,0\rangle$ is dense in $\mathcal{H}_{\text {aniso }}$, we generate the representation of the reduced model by acting only with the subalgebra on the cyclic state. This defines the reduced state space, which is equipped with an inner product by requiring holonomy operators to be unitary. Upon completion, this inner product space defines the Hilbert space of the isotropic model and its quantum representation in agreement with the loop quantization of the classically reduced model. Characteristic properties of the representation are then clearly inherited from that of the less symmetric system, which in some cases is also visible for the reduction from the full theory $[5,18]$.

\subsection{Perturbations}

Dynamical information is obtained by quantizing the Hamiltonian constraints and asking physical states to be annihilated by the resulting operators. Because the constraints contain connection components, and connections are represented via holonomies which shift the labels 
of states as in (25), quantum constraint equations become difference equations [7, 10, 19] for states $|\psi\rangle=\sum_{\mu} \psi_{\mu}|\mu\rangle$ or $|\psi\rangle=\sum_{\mu, \nu} \psi_{\mu, \nu}|\mu, \nu\rangle$ in the triad representation. For large scales, e.g. $\mu \gg 1$ in the isotropic case, the difference equation is well approximated by the Wheeler-DeWitt equation [20] while for small $\mu$ there are crucial differences implying the absence of singularities at the quantum level [11]. In order to see the relation to the WheelerDeWitt equation, one has to assume that the wavefunction does not vary rapidly on small scales, i.e. changes in $\mu$ of the order one, which allows one to Taylor expand the difference operators into differential ones. This limit, however, does not exist for arbitrary states because there is no operator for $\bar{c}$ on $\mathcal{H}_{\text {iso }}$ [12]. Indeed, the Wheeler-DeWitt quantization is based on a representation on the usual Schrödinger Hilbert space $\mathcal{H}_{\mathrm{S}}=L^{2}(\mathbb{R}, \mathrm{d} \bar{c})$ which is inequivalent to the representation on the Bohr Hilbert space used for the loop quantization.

In this paper, we analyse the case of an anisotropic model where, however, difference operators are not expanded for both triad components but only for that corresponding to the anisotropy $p_{\varepsilon}$. We thus assume that the wavefunction does not vary rapidly when $p_{\varepsilon}$ changes and that in an approximate sense an operator for $\varepsilon$ exists. Although we need to assume $p_{\varepsilon}$ to be small, this will not push us into the regime where discreteness is relevant provided that $\bar{p}$ is large. The key additional assumption is that $\varepsilon$ is not too big which now becomes important at the quantum level, and indeed both anisotropy parameters, $p_{\varepsilon}$ and $\varepsilon$, have to be small. (The situation is different from pure isotropy where small $\bar{p}$ usually implies large $\bar{c}$.) When only small $\varepsilon$ are allowed, we do not probe the whole configuration space and do not see its compactness which underlies the Bohr Hilbert space used in homogeneous loop quantizations. As in the large volume limit, we therefore introduce the Schrödinger Hilbert space $\mathcal{H}_{\mathrm{S}}$ for anisotropies, which we do by defining the perturbative Hilbert space

$$
\mathcal{H}_{\text {pert }}:=\mathcal{H}_{\text {iso }} \otimes \mathcal{H}_{\mathrm{S}}
$$

and realizing its dense subset $\mathrm{Cyl}_{\text {iso }} \otimes \mathrm{Cyl}_{\mathrm{S}}$ as a subspace of the dual $\mathrm{Cyl}_{\text {aniso }}^{\star}$. In the Schrödinger Hilbert space we have to choose a suitable dense set $\mathrm{Cyl}_{S}$, which for our purposes will be the set of all functions which are products of a polynomial and a Gaussian. Indeed, Schrödinger states in $\mathrm{Cyl}_{\mathrm{S}}$ can be interpreted as distributions on the Bohr Hilbert space [21] using the antilinear map

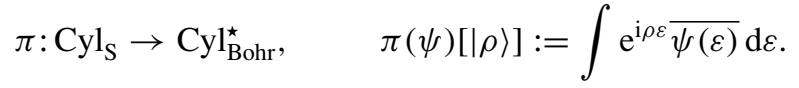

Dual actions of basic operators on those states are given by

$$
\begin{aligned}
\left(\hat{p}_{\varepsilon} \pi(\psi)\right)[|\rho\rangle] & =\pi(\psi)\left[\hat{p}_{\varepsilon}^{\dagger}|\rho\rangle\right]=\frac{1}{6} \gamma \ell_{\mathrm{P}}^{2} \int \rho \mathrm{e}^{\mathrm{i} \rho \varepsilon} \overline{\psi(\varepsilon)} \mathrm{d} \varepsilon=\frac{1}{6} \gamma \ell_{\mathrm{P}}^{2} \int \mathrm{e}^{\mathrm{i} \rho \varepsilon} \cdot \mathrm{i} \frac{\mathrm{d}}{\mathrm{d} \varepsilon} \overline{\psi(\varepsilon)} \mathrm{d} \varepsilon \\
& =\pi\left(-\frac{1}{6} \mathrm{i} \gamma \ell_{\mathrm{P}}^{2} \mathrm{~d} \psi / \mathrm{d} \varepsilon\right)[|\rho\rangle]
\end{aligned}
$$

and

$\left(\mathrm{e}^{\mathrm{i} \tau \varepsilon} \pi(\psi)\right)[|\rho\rangle]=\psi\left[\mathrm{e}^{-\mathrm{i} \tau \varepsilon}|\rho\rangle\right]=\int \mathrm{e}^{\mathrm{i}(\rho-\tau) \varepsilon} \overline{\psi(\varepsilon)} \mathrm{d} \varepsilon=\int \mathrm{e}^{\mathrm{i} \rho \varepsilon} \overline{\mathrm{e}^{\mathrm{i} \tau \varepsilon} \psi(\varepsilon)} \mathrm{d} \varepsilon=\pi\left(\mathrm{e}^{\mathrm{i} \tau \varepsilon} \psi\right)[|\rho\rangle]$.

The first equation shows that the momentum operator is just the derivative operator, as also on the Bohr Hilbert space, while the second shows that $\left(\mathrm{e}^{\mathrm{i} \tau \varepsilon} \pi(\psi)\right)(\varepsilon)=\mathrm{e}^{\mathrm{i} \tau \varepsilon} \psi(\varepsilon)$ where on the Schrödinger Hilbert space we can now take the derivative with respect to $v$. By going to the dual action on the image of $\pi$, we thus obtain a simple multiplication operator for $\varepsilon$ well defined with domain $\pi\left(\mathrm{Cyl}_{\mathrm{S}}\right)$.

In addition, if we choose an appropriate state $\Psi \in \mathrm{Cyl}_{\mathrm{S}}$ we have an embedding id $\otimes \Psi: \mathrm{Cyl}_{\text {iso }} \rightarrow \mathrm{Cyl}_{\text {pert }}$ of isotropic cylindrical states to perturbative cylindrical states where 
anisotropies are small in mean value, but not eliminated exactly. Moreover, since we have to choose a state $\Psi$, which in the above notation is identified with the map $\mathbb{C} \rightarrow \mathrm{Cyl}_{\mathrm{S}}, 1 \mapsto \Psi$, there is no unique canonical embedding. (Coherent states are natural candidates, but unlikely to be preserved dynamically.) In addition to the strict implementation of symmetries $\sigma$ we then have the perturbative implementation $\pi_{\Psi}:=(\star \otimes \pi) \circ(\mathrm{id} \otimes \Psi)$ which are both maps from $\mathrm{Cyl}_{\text {iso }}$ to $\mathrm{Cyl}_{\text {aniso }}^{\star}$ :

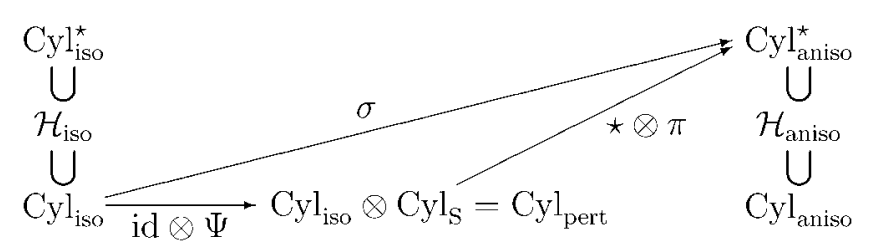

We have the two Gel'fand triples of the exact models on the left and right, with the canonical embedding $\sigma$ of the reduced model through distributions implementing symmetries precisely. In between, we have the perturbative cylindrical space which is connected to the anisotropic side through the antilinear map $\pi$ from $\mathrm{Cyl}_{S}$, combined with the antilinear dualization $\star$ of isotropic states.

In the perturbative sector, non-symmetric degrees of freedom are present but can be arranged to be unexcited at least for initial states of the evolution. In this sense, the situation is similar to the field theory example studied in [22] where non-symmetric field modes are included in their vacuum or other coherent states. In this case, the field theory vacuum is a natural candidate for the analogue of $\Psi$. Perturbative states can then be thought of as states where only the symmetric modes have been excited but non-symmetric ones have been left in their vacua. This is technically similar to our construction of strictly symmetric states at the end of the preceding subsection where we used only symmetric operators in order to generate all states of the symmetric model out of the non-symmetric cyclic state $|0,0\rangle$. Indeed, for background-independent systems in loop quantum gravity the analogue of a ground state is the cyclic state where no geometry at all is excited. Thus, although physically the construction in [22] is closer to the perturbative situation here, technically it is analogous to the situation of the strict model. This illustrates crucial differences in the context of symmetries between quantum field theory on a background and a background-independent quantization of gravity.

We have already discussed the relation between anisotropic operators and isotropic ones for the exact implementation of symmetry. For the perturbative implementation, the same relation exists for operators which do not depend on anisotropies, but we can now also act with other operators as we already saw for $\hat{\varepsilon}$ and $\hat{p}_{\varepsilon}$ in (40) and (39). In general, an anisotropic operator $\hat{O}$ acting on $\mathcal{H}_{\text {aniso }}$ has a dual action on $\star \otimes \pi\left(\mathrm{Cyl}_{\text {pert }}\right)$, but does not necessarily fix this subspace of $\mathrm{Cyl}_{\text {aniso }}^{\star}$. However, it does so perturbatively when we expand it as a sum of operators in the perturbative sector. Choosing, for definiteness, a Gaussian for $\Psi$ we have perturbative states of the form

$$
\psi(A, c)=\mathrm{e}^{\mathrm{i} \bar{v} \bar{c} / 2} \mathrm{e}^{-\left(\varepsilon-\varepsilon_{0}\right)^{2} / 4 \sigma^{2}} \mathrm{e}^{6 \mathrm{i} \varepsilon p_{\varepsilon}^{0} / \gamma \ell_{\mathrm{P}}^{2}}
$$

where $\bar{c}=\frac{1}{3}(2 A+c)$ and $\varepsilon=\frac{1}{3}(A-c)$ are understood as functions of $A$ and $c$. By the chain rule, we then have flux operators

$$
\hat{p}_{A} \psi=-\frac{\mathrm{i}}{2} \gamma \ell_{\mathrm{P}}^{2} \frac{\partial}{\partial A} \psi=-\frac{\mathrm{i}}{6} \gamma \ell_{\mathrm{P}}^{2}\left(2 \frac{\partial}{\partial \bar{c}}+\frac{\partial}{\partial \varepsilon}\right) \psi=\left(\frac{1}{6} \gamma \ell_{\mathrm{P}}^{2} \bar{\nu}+\hat{p}_{\varepsilon}\right) \psi
$$


and

$$
\hat{p}_{c} \psi=-\mathrm{i} \gamma \ell_{\mathrm{P}}^{2} \frac{\partial}{\partial c} \psi=-\frac{\mathrm{i}}{3} \gamma \ell_{\mathrm{P}}^{2}\left(\frac{\partial}{\partial \bar{c}}-\frac{\partial}{\partial \varepsilon}\right) \psi=\left(\frac{1}{6} \gamma \ell_{\mathrm{P}}^{2} \bar{v}-2 \hat{p}_{\varepsilon}\right) \psi .
$$

In composite expressions such as the volume operator one can then expand functions of flux operators in $p_{\varepsilon}$ when they are expressed in the flux representation, and analogously for holonomy operators in the connection representation.

This is most easily done if eigenvalues are already known. For instance, if an operator $\hat{O}$ has eigenstates $|\mu, v\rangle$, we take the eigenvalues $O_{\mu, v}$ and insert

$\mu=\frac{2}{3} \bar{\nu}+4 \gamma^{-1} \ell_{\mathrm{P}}^{-2} p_{\varepsilon}=\frac{2}{3} \bar{\nu}+P, \quad v=\frac{1}{3} \bar{\nu}-4 \gamma^{-1} \ell_{\mathrm{P}}^{-2} p_{\varepsilon}=\frac{1}{3} \bar{\nu}-P$

with dimensionless $P:=4 p_{\epsilon} / \gamma \ell_{\mathrm{P}}^{2}$, which follows from the classical relation (12) or the calculation above. This yields a function $O(\bar{v}, P)$ which we expand in the perturbation $P$,

$$
O(\bar{v}, P)=\sum_{k} O_{\mathrm{iso}}^{(k)}(\bar{v}) P^{k} .
$$

Note that $P$ itself need not be small by our assumptions, which would mean $p_{\varepsilon} \ll \ell_{\mathrm{P}}^{2}$. We have, however, $P \ll \bar{v}$ such that each $O_{\text {iso }}^{(k)}(\bar{v})$ must drop off at least as $\bar{v}^{-k}$. For any fixed $k$, the values $O_{\text {iso }}^{(k)}(\bar{v})$, interpreted as eigenvalues, define an isotropic operator $\hat{O}_{\text {iso }}^{(k)}=\sum_{\bar{v}} O_{\text {iso }}^{(k)}(\bar{v})|\bar{v}\rangle\langle\bar{v}|$ such that $\hat{O}_{\text {iso }}^{(k)}|\bar{v}\rangle:=O_{\text {iso }}^{(k)}(\bar{v})|\bar{v}\rangle$. Thus, we obtain the expansion

$$
\hat{O} \sim \sum_{k} \hat{O}_{\text {iso }}^{(k)} \otimes \hat{P}^{k}
$$

where the right-hand side acts on $\mathcal{H}_{\text {pert }}$. This approximation of operators on a selected class of states implementing small anisotropy is discussed in more detail in appendix A, which can be applied to any Hilbert space $|\bar{\nu}\rangle \otimes \mathcal{H}_{S}$ for fixed $\bar{\nu}$. We understand here always a finite truncation to a given order in $k$, and do not discuss convergence of the expansion in whatever sense. We will later use states $\Psi$ of the above form, assuming $\sigma<\varepsilon_{0} \ll 1$ in order to expand also in $\varepsilon$. This results in similar expansions for operators containing holonomies, such as the Hamiltonian constraint.

\subsection{Hamiltonian constraint}

The Hamiltonian constraint operator for the anisotropic model results from the expression given in [7] after using $A=c_{1}=c_{2}$ and $c=c_{3}$ (as in [7], we ignore an ambiguity parameter $\mu_{0}[12]$ which is of the order one and multiplies connection components in the constraint):

$$
\begin{gathered}
\hat{H}=4 \pi^{-1} \mathrm{i} \gamma^{-3} G^{-1} \ell_{\mathrm{P}}^{-2}\left\{\sin ^{2}\left(\frac{1}{2} A\right) \cos ^{2}\left(\frac{1}{2} A\right) \hat{O}_{c}\right. \\
\left.+2 \sin \left(\frac{1}{2} c\right) \cos \left(\frac{1}{2} c\right) \sin \left(\frac{1}{2} A\right) \cos \left(\frac{1}{2} A\right) \hat{O}_{A}\right\} \\
\hat{O}_{c}:=\sin \left(\frac{1}{2} c\right) \hat{V} \cos \left(\frac{1}{2} c\right)-\cos \left(\frac{1}{2} c\right) \hat{V} \sin \left(\frac{1}{2} c\right)
\end{gathered}
$$

and similarly $\hat{O}_{A}$ by replacing $c$ by $A$, where $\hat{V}$ is the anisotropic volume. Making use of the change of variables (13) one gets

$$
\begin{aligned}
\hat{H}=4 \pi^{-1} \mathrm{i} \gamma^{-3} G^{-1} \ell_{\mathrm{P}}^{-2}\left\{\sin ^{2}\left(\frac{1}{2} \bar{c}+\frac{1}{2} \varepsilon\right) \cos ^{2}\left(\frac{1}{2} \bar{c}+\frac{1}{2} \varepsilon\right) \hat{O}_{c}\right. \\
\left.+2 \sin \left(\frac{1}{2} \bar{c}-\varepsilon\right) \cos \left(\frac{1}{2} \bar{c}-\varepsilon\right) \sin \left(\frac{1}{2} \bar{c}+\frac{1}{2} \varepsilon\right) \cos \left(\frac{1}{2} \bar{c}+\frac{1}{2} \varepsilon\right) \hat{O}_{A}\right\} .
\end{aligned}
$$


Here $\hat{O}_{c}$ and $\hat{O}_{A}$ are more conveniently dealt with by using their action on the $|\mu, v\rangle$ basis, namely,

$$
\begin{aligned}
& \hat{O}_{A}|\mu, v\rangle=\frac{\mathrm{i}}{2}\left(V_{\mu+1, v}-V_{\mu-1, v}\right)|\mu, v\rangle \\
& \hat{O}_{c}|\mu, v\rangle=\frac{\mathrm{i}}{2}\left(V_{\mu, v+1}-V_{\mu, \nu-1}\right)|\mu, v\rangle .
\end{aligned}
$$

Now following (43) we expand $\hat{O}_{A}$ and $\hat{O}_{c}$ up to second order in $p_{\varepsilon}$, using from the exact analysis [7] that

$$
V_{\mu, \nu}=\frac{1}{2}\left(\frac{1}{2} \gamma \ell_{\mathrm{P}}^{2}\right)^{\frac{3}{2}} \sqrt{\mu^{2}|\nu|}=\frac{1}{2}\left(\frac{1}{2} \gamma \ell_{\mathrm{P}}^{2}\right)^{\frac{3}{2}}|\mu| \sqrt{|\nu|} .
$$

Inserting (41), we obtain the eigenvalues

$$
\begin{aligned}
& \left(O_{A}\right)_{\mu(\bar{v}, P), \nu(\bar{v}, P)}=\frac{1}{4} \mathrm{i}\left(\frac{1}{2} \gamma \ell_{\mathrm{P}}^{2}\right)^{3 / 2}\left(\left|\frac{2}{3} \bar{v}+1+P\right|-\left|\frac{2}{3} \bar{v}-1+P\right|\right) \sqrt{\left|\frac{1}{3} \bar{v}-P\right|} \\
& \left(O_{c}\right)_{\mu(\bar{v}, P), \nu(\bar{v}, P)}=\frac{1}{4} \mathrm{i}\left(\frac{1}{2} \gamma \ell_{\mathrm{P}}^{2}\right)^{3 / 2}\left|\frac{2}{3} \bar{v}+P\right|\left(\sqrt{\left|\frac{1}{3} \bar{v}+1-P\right|}-\sqrt{\left|\frac{1}{3} \bar{v}-1-P\right|} .\right.
\end{aligned}
$$

For $P \ll \bar{v}$, the absolute values can be evaluated as

$$
\begin{aligned}
\left|\frac{2}{3} \bar{v}+1+P\right|-\left|\frac{2}{3} \bar{v}-1+P\right| & =\left|\frac{2}{3} \bar{v}+1\right|\left(1+\left(\frac{2}{3} \bar{v}+1\right)^{-1} P\right)-\left|\frac{2}{3} \bar{v}-1\right|\left(1+\left(\frac{2}{3} \bar{v}-1\right)^{-1} P\right) \\
& =\left|\frac{2}{3} \bar{v}+1\right|-\left|\frac{2}{3} \bar{v}-1\right|+\left(\operatorname{sgn}\left(\frac{2}{3} \bar{v}+1\right)-\operatorname{sgn}\left(\frac{2}{3} \bar{v}-1\right)\right) P \\
& =2 \operatorname{sgn}_{3 / 2}(\bar{v})-3 \chi_{3 / 2}(\bar{v}) P,
\end{aligned}
$$

introducing

$$
\begin{aligned}
& \chi_{\delta}(\bar{\nu}):=-\frac{1}{2 \delta}(\operatorname{sgn}(\bar{v}+\delta)-\operatorname{sgn}(\bar{v}-\delta))= \begin{cases}0 & \text { for }|\bar{\nu}| \geqslant \delta \\
\delta^{-1} & \text { for }|\bar{v}|<\delta\end{cases} \\
& \operatorname{sgn}_{\delta}(\bar{\nu}):=\frac{1}{2 \delta}(|\bar{v}+\delta|-|\bar{v}-\delta|)= \begin{cases}1 & \text { for } \bar{v} \geqslant \delta \\
\delta^{-1} \bar{v} & \text { for }|\bar{\nu}|<\delta \\
-1 & \text { for } \bar{v} \leqslant \delta,\end{cases}
\end{aligned}
$$

and

$$
\left|\frac{2}{3} \bar{v}+P\right|=\left|\frac{2}{3} \bar{v}\right|(1+3 P / 2 \bar{v})
$$

while the other expressions are expanded as

and

$$
\sqrt{\left|\frac{1}{3} \bar{v}-P\right|}=\sqrt{\frac{1}{3} \bar{v}}\left(1-\frac{3}{2} P / \bar{v}-\frac{9}{8} P^{2} / \bar{v}^{2}+O\left(P^{3} / \bar{v}^{3}\right)\right)
$$

$$
\begin{aligned}
&\left|\frac{1}{3} \bar{v}+1-P\right|^{1 / 2}-\left|\frac{1}{3} \bar{v}-1-P\right|^{1 / 2} \\
&=\left|\frac{1}{3} \bar{v}+1\right|^{1 / 2} \sqrt{1-\left(\frac{1}{3} \bar{v}+1\right)^{-1} P}-\left|\frac{1}{3} \bar{v}-1\right|^{1 / 2} \sqrt{1-\left(\frac{1}{3} \bar{v}-1\right)^{-1} P} \\
&=\left|\frac{1}{3} \bar{v}+1\right|^{1 / 2}\left(1-\frac{1}{2}\left(\frac{1}{3} \bar{v}+1\right)^{-1} P-\frac{1}{8}\left(\frac{1}{3} \bar{v}+1\right)^{-2} P^{2}\right) \\
&-\left|\frac{1}{3} \bar{v}-1\right|^{1 / 2}\left(1-\frac{1}{2}\left(\frac{1}{3} \bar{v}-1\right)^{-1} P-\frac{1}{8}\left(\frac{1}{3} \bar{v}-1\right)^{-2} P^{2}\right)+O\left(P^{3} / \bar{v}^{3}\right) \\
&=\left|\frac{1}{3} \bar{v}+1\right|^{1 / 2}-\left|\frac{1}{3} \bar{v}-1\right|^{1 / 2}-\frac{1}{2}\left(\left|\frac{1}{3} \bar{v}+1\right|^{-1 / 2} \operatorname{sgn}\left(\frac{1}{3} \bar{v}+1\right)-\left|\frac{1}{3} \bar{v}-1\right|^{-1 / 2}\right. \\
&\left.\times \operatorname{sgn}\left(\frac{1}{3} \bar{v}-1\right)\right) P-\frac{1}{8}\left(\left|\frac{1}{3} \bar{v}+1\right|^{-3 / 2}-\left|\frac{1}{3} \bar{v}-1\right|^{-1 / 2}\right) P^{2}+O\left(P^{3} / \bar{\nu}^{3}\right) \\
&= 2 \sqrt{3} \Delta_{3} \sqrt{|\bar{v}|}-3 \sqrt{3} P \Delta_{3} \frac{\operatorname{sgn}(\bar{v})}{\sqrt{|\bar{v}|}}-\frac{9}{4} \sqrt{3} P^{2} \Delta_{3}|\bar{v}|^{-3 / 2}+O\left(P^{3} / \bar{\nu}^{3}\right)
\end{aligned}
$$


introducing, more generally than before,

$$
\Delta_{\delta} f(\bar{v}):=\frac{1}{2 \delta}(f(\bar{v}+\delta)-f(\bar{v}-\delta))
$$

for any function $f$ which for functions differentiable at large $\bar{v}$ gives $\Delta_{\delta} f(\bar{v}) \sim f^{\prime}(\bar{v})$ for $\bar{v} \gg \delta$.

This results in

$$
\begin{gathered}
\hat{O}_{A}=\frac{1}{4 \sqrt{3}} \mathrm{i}\left(\frac{1}{2} \gamma \ell_{\mathrm{P}}^{2}\right)^{3 / 2} \sqrt{|\hat{\bar{v}}|}\left(2 \operatorname{sgn}_{3 / 2}(\hat{\bar{v}}) \otimes \mathrm{id}-3\left(\chi_{3 / 2}(\hat{\bar{v}})+\hat{\bar{v}}^{-1} \operatorname{sgn}_{3 / 2}(\hat{\bar{v}})\right) \otimes \hat{P}\right. \\
\left.+\frac{9}{2} \hat{\bar{v}}^{-1}\left(\chi_{3 / 2}(\hat{\bar{v}})-\frac{1}{2} \hat{\bar{v}}^{-1} \operatorname{sgn}_{3 / 2}(\hat{\bar{v}})\right) \otimes \hat{P}^{2}+O\left(P^{3} / \bar{v}^{3}\right)\right)
\end{gathered}
$$

and

$$
\begin{gathered}
\hat{O}_{c}=\frac{1}{2 \sqrt{3}} \mathrm{i}\left(\frac{1}{2} \gamma \ell_{\mathrm{P}}^{2}\right)^{3 / 2}|\hat{\bar{v}}|\left(2 \Delta_{3}|\hat{\bar{v}}|^{1 / 2} \otimes \mathrm{id}+3\left(\hat{\bar{v}}^{-1} \Delta_{3}|\hat{\bar{v}}|^{1 / 2}-\Delta_{3}\left(|\hat{\bar{v}}|^{-1 / 2} \operatorname{sgn}(\hat{\bar{v}})\right)\right) \otimes \hat{P}\right. \\
\left.-\frac{9}{2}\left(\hat{\bar{v}}^{-1} \Delta_{3}\left(|\hat{\bar{v}}|^{-1 / 2} \operatorname{sgn}(\hat{\bar{v}})\right)+\frac{1}{2} \Delta_{3}|\hat{\bar{v}}|^{-3 / 2}\right) \otimes \hat{P}^{2}+O\left(P^{3} / \bar{v}^{3}\right)\right) .
\end{gathered}
$$

where $\hat{\bar{v}}=6 \hat{\bar{p}} /\left(\gamma \ell_{\mathrm{P}}^{2}\right)$ and $\hat{P}=4 \hat{p}_{\varepsilon} /\left(\gamma \ell_{\mathrm{P}}^{2}\right)$. From these expressions we can read off the first coefficients $O_{A, \text { iso }}^{(k)}$ and $O_{c, \text { iso }}^{(k)}$.

Note that, while the expressions $\chi_{\delta}, \operatorname{sgn}_{\delta}$ and $\Delta_{\delta}$ can be directly extended to be applied to operators, the expansions are not densely defined on $\mathcal{H}_{\text {pert }}$ because of inverse powers of $\hat{\bar{v}}$. In fundamental expressions where the classical analogue has inverse powers of $\hat{\bar{p}}$, such as matter Hamiltonians, one can still define densely defined operators [23, 24]. Here, however, we started with densely defined operators and obtained inverse powers only after expanding in the perturbative sector. We thus cannot change those terms and make them densely defined. For what follows we can mostly ignore this issue, disregarding states at small $\bar{v}$ on which the operators cannot be applied. We will come back to it in the discussion of singularities in section 5.

Next we must have the action of the several terms to the left of $\hat{O}_{A}$ and $\hat{O}_{c}$ in (45). Up to order $\varepsilon^{3}$ we have

$$
\begin{aligned}
\hat{H}=4 \pi^{-1} \mathrm{i} \gamma^{-3} & G^{-1} \ell_{\mathrm{P}}^{-2}\left[2 \left\{\sin ^{2} \frac{1}{2} \bar{c} \cos ^{2} \frac{1}{2} \bar{c} \otimes \mathrm{id}+\frac{1}{2}\left(-\sin \frac{1}{2} \bar{c} \cos ^{3} \frac{1}{2} \bar{c}+\sin ^{3} \frac{1}{2} \bar{c} \cos \frac{1}{2} \bar{c}\right) \otimes \varepsilon\right.\right. \\
& +\frac{1}{2}\left(-3 \sin ^{2} \frac{1}{2} \bar{c} \cos ^{2} \frac{1}{2} \bar{c}-\cos ^{4} \frac{1}{2} \bar{c}-\sin ^{4} \frac{1}{2} \bar{c}\right) \otimes \varepsilon^{2} \\
& \left.+\frac{1}{12}\left(\sin \frac{1}{2} \bar{c} \cos ^{3} \frac{1}{2} \bar{c}-\sin ^{3} \frac{1}{2} \bar{c} \cos \frac{1}{2} \bar{c}\right) \otimes \varepsilon^{3}+O\left(\varepsilon^{4}\right)\right\} \hat{O}_{A} \\
& +\left\{\sin ^{2} \frac{1}{2} \bar{c} \cos ^{2} \frac{1}{2} \bar{c} \otimes \mathrm{id}+\left(\sin \frac{1}{2} \bar{c} \cos ^{3} \frac{1}{2} \bar{c}-\sin ^{3} \frac{1}{2} \bar{c} \cos \frac{1}{2} \bar{c}\right) \otimes \varepsilon\right. \\
& +\frac{1}{4}\left(-6 \sin ^{2} \frac{1}{2} \bar{c} \cos ^{2} \frac{1}{2} \bar{c}+\cos ^{4} \frac{1}{2} \bar{c}+\sin ^{4} \frac{1}{2} \bar{c}\right) \otimes \varepsilon^{2} \\
& \left.\left.+\frac{2}{3}\left(-\sin \frac{1}{2} \bar{c} \cos ^{3} \frac{1}{2} \bar{c}+\sin ^{3} \frac{1}{2} \bar{c} \cos \frac{1}{2} \bar{c}\right) \otimes \varepsilon^{3}+O\left(\varepsilon^{4}\right)\right\} \hat{O}_{c}\right]
\end{aligned}
$$

From (25), the relevant combinations of sines and cosines on the isotropic basis above have the matrix form

$$
\begin{aligned}
& \left\langle\bar{\nu}\left|\sin ^{2} \frac{1}{2} \bar{c} \cos ^{2} \frac{1}{2} \bar{c}\right| \bar{\mu}\right\rangle=-2^{-4}\left(\delta_{\bar{\nu}, \bar{\mu}+4}-2 \delta_{\bar{\nu}, \bar{\mu}}+\delta_{\bar{\nu}, \bar{\mu}-4}\right), \\
& \left\langle\bar{\nu}\left|\sin \frac{1}{2} \bar{c} \cos ^{3} \frac{1}{2} \bar{c}\right| \bar{\mu}\right\rangle=-2^{-4} \mathrm{i}\left(\delta_{\bar{\nu}, \bar{\mu}+4}+2 \delta_{\bar{\nu}, \bar{\mu}+2}-2 \delta_{\bar{\nu}, \bar{\mu}-2}-\delta_{\bar{\nu}, \bar{\mu}-4}\right), \\
& \left\langle\bar{\nu}\left|\sin ^{3} \frac{1}{2} \bar{c} \cos \frac{1}{2} \bar{c}\right| \bar{\mu}\right\rangle=2^{-4} \mathrm{i}\left(\delta_{\bar{\nu}, \bar{\mu}+4}-2 \delta_{\bar{\nu}, \bar{\mu}+2}+2 \delta_{\bar{\nu}, \bar{\mu}-2}-\delta_{\bar{\nu}, \bar{\mu}-4}\right), \\
& \left\langle\bar{\nu}\left|\sin ^{4} \frac{1}{2} \bar{c}\right| \bar{\mu}\right\rangle=2^{-4}\left(\delta_{\bar{\nu}, \bar{\mu}+4}-4 \delta_{\bar{\nu}, \bar{\mu}+2}+6 \delta_{\bar{\nu}, \bar{\mu}}-4 \delta_{\bar{\nu}, \bar{\mu}-2}+\delta_{\bar{\nu}, \bar{\mu}-4}\right), \\
& \left\langle\bar{v}\left|\cos ^{4} \frac{1}{2} \bar{c}\right| \bar{\mu}\right\rangle=2^{-4}\left(\delta_{\bar{\nu}, \bar{\mu}+4}+4 \delta_{\bar{\nu}, \bar{\mu}+2}+6 \delta_{\bar{\nu}, \bar{\mu}}+4 \delta_{\bar{\nu}, \bar{\mu}-2}+\delta_{\bar{\nu}, \bar{\mu}-4}\right) .
\end{aligned}
$$

So the total action in (54) is given by

$$
\begin{aligned}
\langle\bar{v}|\hat{H}| \bar{\mu}\rangle=\frac{1}{4 \pi} & \mathrm{i} \gamma^{-3} G^{-1} \ell_{\mathrm{P}}^{-2}\left\{\delta_{\bar{\nu}, \bar{\mu}}\left[\left(4-18 \varepsilon^{2}\right) \hat{O}_{A}(\bar{v})+2 \hat{O}_{c}(\bar{v})\right]\right. \\
& +\delta_{\bar{v}, \bar{\mu}+4}\left[\left(-2+2 \mathrm{i} \varepsilon+\varepsilon^{2}\right) \hat{O}_{A}(\bar{v})+\left(-1-2 \mathrm{i} \varepsilon+2 \varepsilon^{2}\right) \hat{O}_{c}(\bar{v})\right] \\
& \left.+\delta_{\bar{v}, \bar{\mu}-4}\left[\left(-2-2 \mathrm{i} \varepsilon+\varepsilon^{2}\right) \hat{O}_{A}(\bar{v})+\left(-1+2 \mathrm{i} \varepsilon+2 \varepsilon^{2}\right) \hat{O}_{c}(\bar{v})\right]+O\left(\varepsilon^{3}\right)\right\}
\end{aligned}
$$


as matrix elements in $\mathcal{H}_{\text {iso }}$ taking operator values in $\mathcal{H}_{\mathrm{S}}$ (for fixed $\bar{v}, \hat{O}_{A}(\bar{v})$ and $\hat{O}_{c}(\bar{v})$ are considered as operators on $|\bar{v}\rangle \otimes \mathcal{H}_{\mathrm{S}} \cong \mathcal{H}_{\mathrm{S}}$ ).

We can act with (56) on a generic state $|s\rangle=\sum_{\nu} s_{v}(\varepsilon)|v\rangle$ and use (52) and (53). For this we write the Hamiltonian in the form

$\langle\bar{v}, \varepsilon|\hat{H}| s\rangle=-\frac{1}{16 \pi} \sqrt{\frac{3}{2}} G^{-1} \gamma^{-3 / 2} \ell_{\mathrm{P}}\left\{\hat{A}_{\bar{\nu}+4} S_{\bar{\nu}+4}(\varepsilon)+\hat{B}_{\bar{\nu}} s_{\bar{\nu}}(\varepsilon)+\hat{C}_{\bar{\nu}-4 S_{\bar{\nu}-4}}(\varepsilon)\right\}$,

where

$$
\begin{aligned}
& \hat{C}_{\bar{\nu}}=-\frac{2}{3}\left(|\bar{\nu}|^{1 / 2} \operatorname{sgn}_{3 / 2}(\bar{\nu})+|\bar{\nu}| \Delta_{3}|\bar{\nu}|^{1 / 2}\right)+\frac{2}{3}\left(|\bar{\nu}|^{1 / 2} \operatorname{sgn}_{3 / 2}(\bar{\nu})-2|\bar{\nu}| \Delta_{3}|\bar{\nu}|^{1 / 2}\right) \mathrm{i} \varepsilon \\
& +\left(\frac{2}{3}|\bar{\nu}|^{1 / 2}\left(\chi_{3 / 2}(\bar{\nu})+\bar{\nu}^{-1} \operatorname{sgn}_{3 / 2}(\bar{\nu})\right)-\operatorname{sgn} \bar{\nu} \Delta_{3}|\bar{\nu}|^{1 / 2}+|\bar{\nu}| \Delta_{3}\left(|\bar{\nu}|^{-1 / 2} \operatorname{sgn} \bar{\nu}\right)\right) \hat{P} \\
& +\frac{3}{2}\left(-|\bar{\nu}|^{-1 / 2} \operatorname{sgn} \bar{v} \chi_{3 / 2}(\bar{\nu})+\frac{1}{2}|\bar{v}|^{-3 / 2} \operatorname{sgn}_{3 / 2}(\bar{v})\right. \\
& \left.+\operatorname{sgn} \bar{\nu} \Delta_{3}\left(|\bar{\nu}|^{-1 / 2} \operatorname{sgn} \bar{\nu}\right)+\frac{1}{2}|\bar{\nu}| \Delta_{3}|\bar{\nu}|^{-3 / 2}\right) \hat{P}^{2}-\left(|\bar{\nu}|^{1 / 2} \chi_{3 / 2}(\bar{\nu})\right. \\
& \left.+|\bar{\nu}|^{-1 / 2} \operatorname{sgn} \bar{\nu} \operatorname{sgn}_{3 / 2}(\bar{\nu})+2 \operatorname{sgn} \bar{\nu} \Delta_{3}|\bar{\nu}|^{1 / 2}-2|\bar{\nu}| \Delta_{3}\left(|\bar{\nu}|^{-1 / 2} \operatorname{sgn} \bar{\nu}\right)\right) \text { i } \varepsilon \hat{P} \\
& +\frac{1}{3}\left(|\bar{v}|^{1 / 2} \operatorname{sgn}_{3 / 2}(\bar{\nu})+4|\bar{\nu}| \Delta_{3}|\bar{v}|^{1 / 2}\right) \varepsilon^{2}+O(3), \\
& \hat{B}_{\bar{\nu}}=\frac{4}{3}\left(|\bar{\nu}|^{1 / 2} \operatorname{sgn}_{3 / 2}(\bar{\nu})+|\bar{\nu}| \Delta_{3}|\bar{\nu}|^{1 / 2}\right)-2\left(|\bar{\nu}|^{1 / 2} \chi_{3 / 2}(\bar{\nu})+|\bar{\nu}|^{-1 / 2} \operatorname{sgn} \bar{\nu} \operatorname{sgn}_{3 / 2}(\bar{\nu})\right. \\
& \left.-\operatorname{sgn} \bar{\nu} \Delta_{3}|\bar{\nu}|^{1 / 2}+|\bar{\nu}| \Delta_{3}\left(|\bar{v}|^{-1 / 2} \operatorname{sgn} \bar{\nu}\right)\right) \hat{P}+3\left(|\bar{\nu}|^{-1 / 2} \operatorname{sgn} \bar{\nu} \chi_{3 / 2}(\bar{\nu})\right. \\
& \left.-\frac{1}{2}|\bar{\nu}|^{-3 / 2} \operatorname{sgn}_{3 / 2}(\bar{\nu})-\operatorname{sgn} \bar{\nu} \Delta_{3}\left(|\bar{\nu}|^{-1 / 2} \operatorname{sgn} \bar{\nu}\right)-\frac{1}{2}|\bar{v}| \Delta_{3}|\bar{\nu}|^{-3 / 2}\right) \hat{P}^{2} \\
& -6|\bar{\nu}|^{1 / 2} \operatorname{sgn}_{3 / 2}(\bar{\nu}) \varepsilon^{2}+O(3)
\end{aligned}
$$

and $\hat{A}_{\bar{\nu}}=\overline{\hat{C}}_{\bar{\nu}}$. Note that this is not the adjoint of $\hat{C}_{\bar{\nu}}$ but the complex conjugate (acting only on the numerical coefficients, not on possible factors of the imaginary unit in a derivative representation of $\hat{P}$ ). Complex conjugation and adjoint are not the same in this case because of the $\hat{\varepsilon} \hat{P}$ term. Also the whole constraint operator is not self-adjoint since we started with a non-symmetric ordering with holonomy operators to the left.

\section{Evolution}

The constraint equation given by the operator (57), i.e. requiring physical states to be annihilated by the constraint operator, results in a difference-differential equation for $s_{n}(\varepsilon)$ which can be interpreted as an evolution equation in the volume $\bar{v}$ as internal time. This equation is difference-differential because we keep the underlying discreteness from quantum geometry in the isotropic variable, but treat the anisotropy perturbatively. Also in isotropic models one can have difference-differential equations if there is a matter degree of freedom such as a scalar, but the crucial difference here is that there are differential operators at all levels of the difference equation and in particular the leading ones. This has implications for the recurrence scheme since those operators have to be inverted in some manner. We perform here only a basic analysis to check if the scheme has satisfactory properties concerning evolution and relation to classical behaviour.

We focus on the stability issue for which in our context three different notions occur. First, in the perturbative setting stability is already relevant at the classical level where one needs to make sure that anisotropies stay small enough for sufficiently long time. As can be seen from the discussion leading to (22), this can be ensured for some range of evolution at least in the vacuum case for our situation. The second notion is stability at the quantum level in the sense 
of [25], requiring that there are no exponentially growing solutions to the difference equation. This will be the focus in the following sections. Finally, also numerical stability issues can arise when difference or difference-differential equations are solved numerically.

We consider the evolution equation (57) without matter for large $\bar{v}$, for which the constraint equation reduces to

$$
\begin{aligned}
\frac{-\sqrt{3} \ell_{\mathrm{P}}}{2^{3 / 2} \kappa \gamma^{3 / 2}}[( & \left.-\frac{4}{3}+\frac{4}{3} \mathrm{i} \varepsilon+\frac{2}{3} \varepsilon^{2}-\frac{6}{\bar{v}} \mathrm{i} \varepsilon \hat{P}+\frac{3}{2 \bar{v}^{2}} \hat{P}^{2}\right) s_{\bar{v}+4}(\varepsilon) \\
& +\left(-\frac{4}{3}-\frac{4}{3} \mathrm{i} \varepsilon+\frac{2}{3} \varepsilon^{2}+\frac{6}{\bar{v}} \mathrm{i} \varepsilon \hat{P}+\frac{3}{2 \bar{v}^{2}} \hat{P}^{2}\right) s_{\bar{v}-4}(\varepsilon) \\
& \left.+\left(\frac{8}{3}-\frac{2}{\bar{v}} \hat{P}-12 \varepsilon^{2}-\frac{3}{\bar{v}^{2}} \hat{P}^{2}\right) s_{\bar{v}}(\varepsilon)\right]=0
\end{aligned}
$$

Now we propose a restricted function space for the solutions of the form

$$
s_{\bar{v}}(\varepsilon)=p_{\bar{\nu}}^{(k)}(\varepsilon) \mathrm{e}^{-\left(\varepsilon-\varepsilon_{0}\right)^{2} / 4 \sigma^{2}},
$$

where $p_{\bar{\nu}}^{(k)}$ are complex-valued polynomials of order $k$. Since we expanded the Hamiltonian constraint to second order, we also should disregard terms of higher order in the solutions and work with $k=2$. Then,

$$
p_{\bar{\nu}}^{(2)}(\varepsilon)=A_{0}(\bar{\nu})+A_{1}(\bar{v}) \varepsilon+A_{2}(\bar{v}) \varepsilon^{2}
$$

where $A_{0}, A_{1}$ and $A_{2}$ are complex-valued functions of the discrete time $\bar{v}$ only, to be determined from initial values through the difference equation. This class of functions is general enough for our purposes because, even though $\varepsilon_{0}$ and $\sigma$ are fixed, the Gaussian can be deformed and spread if $A_{1}$ and $A_{2}$ grow as functions of $\bar{v}$. Moreover, to the given order of expansion also nonzero expectation values for $\hat{p}_{\varepsilon}$ are allowed through complex-valued $A_{1}$ and $A_{2}$. For a Gaussian state peaked at anisotropy $p_{\varepsilon}^{0}$ we need a phase factor $\mathrm{e}^{6 \mathrm{i} \varepsilon p_{\varepsilon}^{0} / \gamma \ell_{\mathrm{P}}^{2}}$ which for small enough $p_{\varepsilon}^{0}$ can be expanded, only contributing imaginary parts to $A_{1}$ and $A_{2}$. For this, we need to assume $p_{\varepsilon}^{0} \ll \ell_{\mathrm{P}}^{2}$ which is possible but not guaranteed by our general perturbation assumptions. Still, for the tests we are going to perform such small values are sufficient. For more detailed aspects one has to generalize the allowed class of functions.

Computing (60) using $\hat{P}=-\frac{2}{3} \mathrm{id} / \mathrm{d} \varepsilon$ and (61), (62) for large $\bar{v}$, and collecting terms of the same order in $\varepsilon$ we obtain

$$
\begin{aligned}
A_{0}(\bar{v}+4)\left(2 \sigma^{2}\right. & \left.-8 \bar{v}^{2} \sigma^{4}-\varepsilon_{0}^{2}\right)-2 A_{0}(\bar{v})\left(2 \sigma^{2}-8 \bar{v}^{2} \sigma^{4}-2 \mathrm{i} \bar{v} \sigma^{2} \varepsilon_{0}-\varepsilon_{0}^{2}\right) \\
& +A_{0}(\bar{v}-4)\left(2 \sigma^{2}-8 \bar{v}^{2} \sigma^{4}-\varepsilon_{0}^{2}\right)+A_{1}(\bar{v}+4)\left(-4 \sigma^{2} \varepsilon_{0}\right) \\
& -2 A_{1}(\bar{v})\left(-4 \mathrm{i} \bar{v} \sigma^{4}-4 \sigma^{2} \varepsilon_{0}\right)+A_{1}(\bar{v}-4)\left(-4 \sigma^{2} \varepsilon_{0}\right) \\
& +A_{2}(\bar{v}+4)\left(-8 \sigma^{4}\right)-2 A_{2}(\bar{v})\left(-8 \sigma^{4}\right)+A_{2}(\bar{v}-4)\left(-8 \sigma^{4}\right)=0
\end{aligned}
$$

from leading order in $\varepsilon$,

$$
\begin{aligned}
A_{0}(\bar{v}+4)\left(8 \mathrm{i} \bar{\nu}^{2}\right. & \left.\sigma^{4}-12 \bar{v} \sigma^{2} \varepsilon_{0}+2 \varepsilon_{0}\right)-2 A_{0}(\bar{v})\left(-2 \mathrm{i} \bar{\nu} \sigma^{2}+2 \varepsilon_{0}\right) \\
& +A_{0}(\bar{v}-4)\left(-8 \mathrm{i} \bar{\nu}^{2} \sigma^{4}+12 \bar{v} \sigma^{2} \varepsilon_{0}+2 \varepsilon_{0}\right) \\
& +A_{1}(\bar{v}+4)\left(6 \sigma^{2}-24 \bar{v} \sigma^{4}-8 \bar{v}^{2} \sigma^{4}-\varepsilon_{0}^{2}\right) \\
& -2 A_{1}(\bar{v})\left(6 \sigma^{2}-8 \bar{v}^{2} \sigma^{4}-\varepsilon_{0}^{2}-2 \mathrm{i} \bar{\nu} \sigma^{2} \varepsilon_{0}\right) \\
& +A_{1}(\bar{v}-4)\left(6 \sigma^{2}+24 \bar{v} \sigma^{4}-8 \bar{v}^{2} \sigma^{4}-\varepsilon_{0}^{2}\right) \\
& +A_{2}(\bar{v}+4)\left(-8 \sigma^{2} \varepsilon_{0}\right)-2 A_{2}(\bar{v})\left(-8 \mathrm{i} \bar{v} \sigma^{4}-8 \sigma^{2} \varepsilon_{0}\right) \\
& +A_{2}(\bar{v}-4)\left(-8 \sigma^{2} \varepsilon_{0}\right)=0
\end{aligned}
$$


from linear order, and

$$
\begin{aligned}
A_{0}(\bar{v}+4)(-1 & \left.+12 \bar{v} \sigma^{2}+4 \bar{v}^{2} \sigma^{4}\right)-2 A_{0}(\bar{v})\left(-1+36 \bar{v}^{2} \sigma^{4}\right) \\
& +A_{0}(\bar{v}-4)\left(-1-12 \bar{v} \sigma^{2}+4 \bar{v}^{2} \sigma^{4}\right) \\
& +A_{1}(\bar{v}+4)\left(8 \mathrm{i} \bar{v}^{2} \sigma^{4}+2 \varepsilon_{0}-12 \bar{v} \sigma^{2} \varepsilon_{0}\right)-2 A_{1}(\bar{v})\left(2 \mathrm{i} \bar{v} \sigma^{2}+2 \varepsilon_{0}\right) \\
& +A_{1}(\bar{v}-4)\left(-8 \mathrm{i} \bar{v}^{2} \sigma^{4}+2 \varepsilon_{0}+12 \bar{v} \sigma^{2} \varepsilon_{0}\right) \\
& +A_{2}(\bar{v}+4)\left(10 \sigma^{2}-48 \bar{v} \sigma^{4}-8 \bar{v}^{2} \sigma^{4}-\varepsilon_{0}^{2}\right) \\
& -2 A_{2}(\bar{v})\left(10 \sigma^{2}-8 \bar{v}^{2} \sigma^{4}-2 \mathrm{i} \bar{v} \sigma^{2} \varepsilon_{0}-\varepsilon_{0}^{2}\right) \\
& +A_{2}(\bar{v}-4)\left(10 \sigma^{2}+48 \bar{v} \sigma^{4}-8 \bar{v}^{2} \sigma^{4}-\varepsilon_{0}^{2}\right)=0
\end{aligned}
$$

from quadratic order. As the equations are quite complicated, we discuss here mainly their approximation for large volume which can already be used to see relations to the classical behaviour.

\subsection{Asymptotic $\bar{v}$ solution}

While the perturbation scheme does not require a relation between the magnitudes of $\bar{v}$ and $\varepsilon_{0}$ or $\sigma \sim \varepsilon_{0} \ll 1$, for a given choice of $\varepsilon_{0}$ and $\sigma$ consistent with the perturbation assumptions we can look at the asymptotic form of the equations where only highest powers of $\bar{v}$ are used. For the second-order equations, this requires $\bar{v} \gg \sigma^{-2}$ which is our assumption for the asymptotic regime. Simplifying the equations then leads to

$$
\begin{aligned}
& A_{0}(\bar{v}+4)-2 A_{0}(\bar{v})+A_{0}(\bar{v}-4)=0 \\
& A_{1}(\bar{v}+4)-2 A_{1}(\bar{v})+A_{1}(\bar{v}-4)=\mathrm{i}\left[A_{0}(\bar{v}+4)-A_{0}(\bar{v}-4)\right], \\
& \begin{aligned}
& A_{2}(\bar{v}+4)-2 A_{2}(\bar{v})+A_{2}(\bar{v}-4)=\frac{1}{2}\left[A_{0}(\bar{v}+4)-18 A_{0}(\bar{v})+A_{0}(\bar{v}-4)\right] \\
&+\mathrm{i}\left[A_{1}(\bar{v}+4)-A_{1}(\bar{v}-4)\right] .
\end{aligned}
\end{aligned}
$$

We can already observe that, in contrast to (63), these equations are independent of the value of $\varepsilon_{0}$ in complete analogy to our previous observation for the asymptotic classical solution (22). Also, the equations have imaginary terms which are necessary for $p_{\varepsilon}$ to change in internal time (in the $\varepsilon$ representation chosen here, $p_{\varepsilon}$ enters through the phase of the wavefunction). Even if $p_{\varepsilon}^{0}$ is initially zero, i.e. $A_{0}, A_{1}$ and $A_{2}$ are all real, the wavefunction must become complex after some steps such that anisotropy also in the triad arises automatically.

Before solving (66) we generalize it to allow dust-like matter (matter Hamiltonian independent of $\bar{v}$ ) such that the middle coefficients on the left-hand sides of the equations are $2 \rho$ with a constant $\rho \leqslant 1$, reproducing the vacuum case above for $\rho=1$. We then put the system in a first-order form, considering solutions only on the lattice $4 \mathbb{Z}$ which is sufficient to see the behaviour of long-time evolution. Accordingly, we introduce the vector

$$
\mathbf{v}(\bar{v}+4)=\left(\begin{array}{c}
A_{0}(\bar{v}+4) \\
A_{1}(\bar{v}+4) \\
A_{2}(\bar{v}+4) \\
A_{0}(\bar{v}) \\
A_{1}(\bar{v}) \\
A_{2}(\bar{v})
\end{array}\right) .
$$

With this definition we can cast (66) in a first-order linear matrix equation (of step-size four):

$$
M \mathbf{v}(\bar{v}+4)+N \mathbf{v}(\bar{v})=\mathbf{0} \quad \text { or } \quad \mathbf{v}(\bar{v}+4)+Q \mathbf{v}(\bar{v})=\mathbf{0},
$$


where the matrix $Q$ is

$$
Q=\left(\begin{array}{cccccc}
-2 \rho & 0 & 0 & 1 & 0 & 0 \\
-2 \mathrm{i} \rho & -2 \rho & 0 & 2 \mathrm{i} & 1 & 0 \\
9+\rho & 2 \mathrm{i} \rho & -2 \rho & -2 & 2 \mathrm{i} & 1 \\
-1 & 0 & 0 & 0 & 0 & 0 \\
0 & -1 & 0 & 0 & 0 & 0 \\
0 & 0 & -1 & 0 & 0 & 0
\end{array}\right)
$$

It is possible to bring this matrix to its Jordan canonical form (see appendix B) $J=S^{-1} Q S$ where

$$
J=\left(\begin{array}{cccccc}
\lambda_{1} & 1 & 0 & 0 & 0 & 0 \\
0 & \lambda_{1} & 1 & 0 & 0 & 0 \\
0 & 0 & \lambda_{1} & 0 & 0 & 0 \\
0 & 0 & 0 & \lambda_{2} & 1 & 0 \\
0 & 0 & 0 & 0 & \lambda_{2} & 1 \\
0 & 0 & 0 & 0 & 0 & \lambda_{2}
\end{array}\right)
$$

Here, $\lambda_{1,2}= \pm \sqrt{\rho^{2}-1}-\rho$ are the two eigenvalues of $Q$ with multiplicity three (which degenerate further in the vacuum case $\rho=1$ ).

From here we can obtain the solution starting from initial conditions in (67):

$$
\mathbf{v}\left(\bar{v}_{0}+4 k\right)=Q^{k} \mathbf{v}\left(\bar{v}_{0}\right) .
$$

We obtain

$$
Q^{k}=S J^{k} S^{-1},
$$

where $J$ is the Jordan form for $Q$ above and each one of its block powers is

$$
J_{i}^{k}=\sum_{j=0}^{2}\left(\begin{array}{l}
k \\
j
\end{array}\right) \lambda_{i}^{k-j} N^{j}
$$

$N$ is the off-diagonal term in $J$.

We obtain the transition matrix $S$ by computing the generalized eigenvectors associated with $\lambda_{1,2}$, which we do not write down; however we write the general $k$ power of $J$ above:

$$
\boldsymbol{J}^{k}=\left(\begin{array}{cccccc}
\lambda_{1}^{k} & k \lambda_{1}^{k-1} & \left(\begin{array}{c}
k \\
2
\end{array}\right) \lambda_{1}^{k-2} & 0 & 0 & 0 \\
0 & \lambda_{1}^{k} & k \lambda_{1}^{k-1} & 0 & 0 & 0 \\
0 & 0 & \lambda_{1}^{k} & 0 & 0 & 0 \\
0 & 0 & 0 & \lambda_{2}^{k} & k \lambda_{2}^{k-1} & \left(\begin{array}{c}
k \\
2
\end{array}\right) \lambda_{2}^{k-2} \\
0 & 0 & 0 & 0 & \lambda_{2}^{k} & k \lambda_{2}^{k-1} \\
0 & 0 & 0 & 0 & 0 & \lambda_{2}^{k}
\end{array}\right) .
$$

From this the solution for the $A$ functions is

$$
\begin{aligned}
A_{0}\left(\bar{v}_{0}+4 k\right)= & \frac{1}{2}\left(\rho^{2}-1\right)^{-1 / 2}\left(\left(-\lambda_{1}^{k} \lambda_{2}+\lambda_{1} \lambda_{2}^{k}\right) A_{0}\left(\bar{v}_{0}\right)+\left(\lambda_{2}^{k}-\lambda_{1}^{k}\right) A_{0}\left(\bar{v}_{0}-4\right)\right), \\
A_{1}\left(\bar{v}_{0}+4 k\right)= & \frac{1}{2}\left(\rho^{2}-1\right)^{-1 / 2}\left(\left(-\lambda_{1}^{k} \lambda_{2}+\lambda_{1} \lambda_{2}^{k}\right) A_{1}\left(\bar{v}_{0}\right)-\mathrm{i} k\left(-\lambda_{1}^{2} \lambda_{2}^{k-1}+\lambda_{1}^{k-1} \lambda_{2}^{2}\right) A_{0}\left(\bar{v}_{0}\right)\right. \\
& \left.+\mathrm{i}\left(\lambda_{2}^{k}-\lambda_{1}^{k}-k \lambda_{1}^{k-1} \lambda_{2}+k \lambda_{2}^{k-1} \lambda_{1}\right) A_{0}\left(\bar{v}_{0}-4\right)\left(\lambda_{2}^{k}-\lambda_{1}^{k}\right) A_{1}\left(\bar{v}_{0}-4\right)\right)
\end{aligned}
$$


and

$$
\begin{aligned}
& A_{2}\left(\bar{v}_{0}+4 k\right)=\frac{1}{4}\left[\frac{9}{\left(\rho^{2}-1\right)^{3 / 2}}\left(\lambda_{1}^{k}-\lambda_{2}^{k}\right)\right. \\
& +k \lambda_{1}^{k-1}\left(\frac{-9-2 \rho+18 \rho^{2}+2 \rho^{3}}{\rho^{2}-1}+\frac{-1+18 \rho+2 \rho^{2}}{\sqrt{\rho^{2}-1}}\right) \\
& +\left(\begin{array}{l}
k \\
2
\end{array}\right) \frac{2 \lambda_{1}^{k-2}}{\rho^{2}-1}\left(1-5 \rho^{2}+4 \rho^{4}-3 \rho \sqrt{\rho^{2}-1}+4 \rho^{3} \sqrt{\rho^{2}-1}\right) \\
& +k \lambda_{2}^{k-1}\left(\frac{-9-2 \rho+18 \rho^{2}+2 \rho^{3}}{\rho^{2}-1}+\frac{-1+18 \rho+3 \rho^{2}+18 \rho^{3}-2 \rho^{4}}{\left(\rho^{2}-1\right)^{3 / 2}}\right) \\
& \left.+\left(\begin{array}{l}
k \\
2
\end{array}\right) \lambda_{2}^{k-2}\left(\frac{2-12 \rho^{2}+18 \rho^{4}-8 \rho^{6}}{\left(\rho^{2}-1\right)^{2}}+\frac{6 \rho-14 \rho^{3}+8 \rho^{5}}{\left(\rho^{2}-1\right)^{3 / 2}}\right)\right] A_{0}\left(\bar{v}_{0}\right) \\
& -\frac{\mathrm{i} k}{2}\left(\frac{\lambda_{1}^{k-1} \lambda_{2}^{2}-\lambda_{2}^{k-1} \lambda_{1}}{\sqrt{\rho^{2}-1}}\right) A_{1}\left(\bar{v}_{0}\right)+\frac{1}{2}\left(\frac{-\lambda_{1}^{k} \lambda_{2}+\lambda_{2}^{k} \lambda_{1}}{\sqrt{\rho^{2}-1}}\right) A_{2}\left(\bar{v}_{0}\right) \\
& +\frac{1}{4}\left[\lambda_{1}^{k} \frac{1-9 \rho+\rho^{2}}{\left(\rho^{2}-1\right)^{3 / 2}}+\lambda_{2}^{k} \frac{1+9 \rho-\rho^{2}}{\left(\rho^{2}-1\right)^{3 / 2}}\right. \\
& +3 k\left(-\lambda_{1}^{k-1}\left\{1+\frac{3 \rho}{\rho^{2}-1}+\frac{3+\rho}{\sqrt{\rho^{2}-1}}\right\}+\lambda_{2}^{k-1}\left\{-1-\frac{3 \rho}{\rho^{2}-1}+\frac{3+\rho}{\sqrt{\rho^{2}-1}}\right\}\right) \\
& +\left(\begin{array}{l}
k \\
2
\end{array}\right)\left(\lambda_{1}^{k-2}\left\{4 \rho+2 \frac{2 \rho^{2}-1}{\sqrt{\rho^{2}-1}}\right\}\right. \\
& \left.\left.+\lambda_{2}^{k-2}\left\{\frac{4 \rho}{(\rho+1)^{2}}-2 \frac{2 \rho^{2}-1}{(\rho+1)^{3 / 2} \sqrt{\rho-1}}\right\}\right)\right] A_{0}\left(\bar{v}_{0}-4\right) \\
& +\frac{\mathrm{i}}{2}\left(\frac{\lambda_{2}^{k}-\lambda_{1}^{k}}{\left(\rho^{2}-1\right)^{\frac{3}{2}}}-k \frac{\lambda_{1}^{k-1} \lambda_{2}-\lambda_{1} \lambda_{2}^{k-1}}{\sqrt{\rho^{2}-1}}\right) A_{1}\left(\bar{v}_{0}-4\right)+\frac{\lambda_{2}^{k}-\lambda_{1}^{k}}{2 \sqrt{\rho^{2}-1}} A_{2}\left(\bar{v}_{0}-4\right) \text {. }
\end{aligned}
$$

This solution is valid only for $|\rho| \neq 1$, for otherwise the eigenvalues would be completely degenerate.

So we have obtained the solution to the evolution equation in the asymptotic $\bar{v}$ regime. We should note that the solutions grow as powers of the order $k$ (no larger than second) as a consequence of degeneracy, although the leading coefficient $A_{0}$ is bounded.

\subsection{Inclusion of matter}

One can hope that matter terms remove the degeneracy present for the case for pure gravity, which similarly happens in the isotropic model (see [11] for an explicit isotropic vacuum solution which is also unbounded). As the previous discussion shows, however, this is not the case for dust which has a constant diagonal Hamiltonian, $\hat{H}=E \mathbb{I}$.

As the next simple possibility we can consider a cosmological constant term

$$
\hat{H}_{\text {matter }}=\Lambda \hat{V} .
$$


Using (48) the evolution equation for large $\bar{v}(60)$ modifies to

$$
\begin{aligned}
& \left(-\frac{4}{3}+\frac{4}{3} \mathrm{i} \varepsilon+\frac{2}{3} \varepsilon^{2}-\frac{6}{\bar{v}} \mathrm{i} \varepsilon \hat{P}+\frac{3}{2 \bar{v}^{2}} \hat{P}^{2}\right) s_{\bar{\nu}+4}+\left(-\frac{4}{3}-\frac{4}{3} \mathrm{i} \varepsilon+\frac{2}{3} \varepsilon^{2}+\frac{6}{\bar{v}} \mathrm{i} \varepsilon \hat{P}+\frac{3}{2 \bar{v}^{2}} \hat{P}^{2}\right) s_{\bar{v}-4} \\
& +\left(\frac{8}{3}-\frac{2}{\bar{v}} \hat{P}-12 \varepsilon^{2}-\frac{3}{\bar{v}^{2}} \hat{P}^{2}\right) s_{\bar{\nu}}=-\frac{4}{3} \pi G \gamma^{3} \Lambda\left(\frac{2 \bar{\nu}^{3 / 2}}{3}-\frac{9}{4 \sqrt{\bar{\nu}}} \hat{P}^{2}\right) s_{\bar{\nu}} .
\end{aligned}
$$

With the same ansatz (61), (62) the right-hand side of each equation becomes

$\frac{\bar{v}^{3 / 2}}{6}\left[A_{0}(\bar{v})\left(54 \sigma^{2}+32 \bar{v}^{2} \sigma^{4}-27 \varepsilon_{0}^{2}\right)+A_{1}(\bar{v})\left(-108 \sigma^{2} \varepsilon_{0}\right)+A_{2}(\bar{v})\left(-216 \sigma^{4}\right)\right]$

for (63),

$$
\frac{\bar{v}^{3 / 2}}{6}\left[A_{0}(\bar{v})\left(54 \varepsilon_{0}\right)+A_{1}(\bar{v})\left(162 \sigma^{2}+32 \bar{v}^{2} \sigma^{4}-27 \varepsilon_{0}^{2}\right)+A_{2}(\bar{v})\left(-216 \sigma^{2} \varepsilon_{0}\right)\right]
$$

for (64) and

$$
\frac{\bar{v}^{3 / 2}}{6}\left[A_{0}(\bar{v})(-27)+A_{1}(\bar{v})\left(54 \varepsilon_{0}\right)+A_{2}(\bar{v})\left(270 \sigma^{2}+32 \bar{\nu}^{2} \sigma^{4}-27 \varepsilon_{0}^{2}\right)\right]
$$

for $(65)$.

It is easier to check the degeneracy in the asymptotic $\bar{v}$ regime: in this case the righthand sides of (66) acquire an extra term $\frac{2^{7}}{9} \pi G \gamma^{3} \Lambda \bar{\nu}^{3 / 2} A_{i}(\bar{v})$, respectively. Coefficients in the asymptotic difference equation are thus no longer constant and we do not find explicit solutions. Still, around any fixed $\bar{v}$ eigenvalues remain degenerate.

\subsection{Large $\bar{v}$ behaviour}

Rather than looking at more involved matter choices, we now use only large $\bar{v}$ and keep next order terms in $\bar{v}^{-1}$. This gives rise to more complicated correction terms in (63) compared to the asymptotic equations, changing all coefficients. Rather than trying to solve this secondorder system of difference equations, we perform a local stability analysis around large fixed $\bar{v}$ similar to that in the previous section.

To this end we put the system (60) again in a first-order form (67), where now the matrices $\mathbf{M}, \mathbf{N}, \mathbf{Q}$ are the functions of $\left(\bar{\nu}, \varepsilon_{0}, \sigma\right)$. In this case the stability of the system is obtained if and only if the module of the eigenvalues of the matrix $\mathbf{Q}$ above are less or equal to 1 . However, its characteristic polynomial is of order six and, recalling the form of (63), each one of its coefficients is a very complex function of $\left(\bar{v}, \varepsilon_{0}, \sigma\right)$. Therefore, we perform a numerical analysis to see the behaviour of each one of the coefficient functions $A_{i}(\bar{v})$ in $(62)$.

The characteristic polynomial is of the form

$$
\lambda^{6}+h_{5} \lambda^{5}+h_{4} \lambda^{4}+h_{3} \lambda^{3}+h_{2} \lambda^{2}+h_{1} \lambda+h_{0}=0, \quad h_{j}=h_{j}\left(\bar{\nu}, \varepsilon_{0}, \sigma\right), \quad j=0, \ldots, 5
$$

which one can solve numerically to find choices of $\varepsilon_{0}$ and $\sigma$ where all six roots have an absolute value less than or equal to 1 .

\section{Perturbations and the singularity}

When perturbing in basic variables, the expansion is valid only for $p_{\varepsilon} \ll \bar{p}$ and thus breaks down very close to the classical singularity. Indeed, the main aim for studying perturbations is to understand effects in structure formation where one needs at least 
perturbative inhomogeneities for which anisotropies serve as a model. This is usually modelled in semiclassical regimes, in an inflationary phase or sometimes through a bounce at relatively large volume, far away from the Planck scale. It is nevertheless instructive to discuss implications for the singularity issue itself close to vanishing $\bar{p}$, as they might appear in a perturbative setting.

In the asymptotic $\bar{v}$ equation (66) we saw that the relevant matrix $M$ in (67) at highest order of the difference equation is always invertible such that evolution cannot break down in this regime. But this is not guaranteed at small $\bar{v}$ where a breakdown of evolution can occur even at $\bar{v}>0$ where one would not expect singularities classically. There are even divergences in coefficients of the difference equation such as in $\hat{C}_{\bar{v}}$ for $\bar{v}=3$ coming, e.g., from $\Delta_{3}|\bar{\nu}|^{-3 / 2}$. This is certainly a small value for $\bar{\nu}$, but note that $p_{\varepsilon}$ can be small compared to $\bar{p}$ even for $\bar{v}$ of the order one which perturbatively is consistent with using the fact that the perturbative operator $p_{\varepsilon}$ has a continuous spectrum. As discussed earlier, small $p_{\varepsilon}$ do not automatically bring us into discrete regimes, unlike small $\bar{p}$. One can thus set up the perturbative evolution such that all assumptions for the perturbation scheme are satisfied, and yet the evolution breaks down at nonzero volume. (Starting at large volume, the perturbative approximation is expected to break down much before the classical singularity is reached. But generically, a breakdown of evolution cannot be ruled out since there are initial conditions for which the evolution breaks down before the perturbative scheme ceases to make sense.)

There is thus no removal of singularities in the perturbative model. Even worse, the perturbative equation could make us believe that the non-singular evolution of the isotropic model is very special and could not extend to less symmetric models, owing to the fact that for $\varepsilon=P=0$ all divergent coefficients disappear. In such a situation, it is necessary to use the full model from which the perturbative equation is derived in order to see whether or not it breaks down. As it turns out, for loop quantum cosmology the evolution is non-singular even for anisotropic models, in contrast to the perturbative appearance.

A different source for a breakdown of evolution appears because coefficients of the difference equation are now differential operators on the perturbative degree of freedom. These operators must be inverted to proceed with the evolution, which is not generically possible and may require special boundary conditions. Note that, if a physical inner product is not known, even generic invertibility would not be enough: one needs to make sure that any initial state can be evolved without breakdown to conclude singularity freedom. Even if almost all initial values would lead to non-singular evolution, all of them could be ruled out by the physical inner product (see also [6]). We do not discuss this in detail here but only conclude that, while the fully quantized anisotropic model is non-singular, there is no such statement in the perturbative quantization.

It is interesting to compare this situation with recent results in string theory [26] where inhomogeneities on a background are seen to prevent the occurrence of a bounce instead of a singularity (which would otherwise be possible in the corresponding homogeneous model). This looks similar to our perturbative quantization of anisotropies on an isotropic background, even though the models and techniques are certainly very different. In contrast, non-perturbative background-independent models studied so far are non-singular, including inhomogeneous ones which classically have local physical degrees of freedom [27].

There is a further disadvantage of using perturbative or semiclassical degrees of freedom in order to discuss the singularity issue, as for instance suggested in [28]. Such a perturbative treatment is unlikely to remain valid close to a singularity where potentially all degrees of freedom can be excited strongly. Perturbations certainly allow one to include all degrees of freedom, which is important for phenomenology, but properties of the singularity can be extremely blurred as we have seen here. In contrast, symmetric models completely remove 
many degrees of freedom which may have to be reinserted later on when discussing their effect on evolution. But symmetric models themselves are often classically singular, and those singularities can be studied by quantizations of models. With the models now available, the most characteristic types of classical singularities can be studied also at the quantum level. This can then show how classical singularities can be resolved by quantization, and there is indeed a general mechanism in loop quantum gravity.

While the fundamental singularity issue concerning the extension of solutions to the Hamiltonian constraint equation cannot be analysed perturbatively, this is still possible for phenomenological models of bounces as studied in [29] and used for the construction of oscillatory universe models in [30]. Such bounces need to happen sufficiently far above the Planck scale in order to ensure the validity of effective equations [31], and thus avoid a potential breakdown of the perturbative discrete evolution. This will also be true for perturbative analyses of inhomogeneities. Also at the inhomogeneous level, one can employ approximate but non-perturbative methods in the spirit of the BKL picture [32]. In contrast to perturbative schemes, this can then also be applied to the fundamental singularity issue, as done in the loop quantum gravity context with preliminary investigations in [33].

\section{Conclusions}

We have mainly illustrated the reduction procedure to symmetric situations available for loop quantum gravity, and contrasted it with a new method for perturbative quantum degrees of freedom in the case of anisotropy. We consider this mainly as a model situation for the cosmologically more interesting but technically much more complicated case of perturbative inhomogeneities. This already allowed us to draw some cautionary conclusions for the singularity problem when discussed in a perturbative setting, as well as for the relation between symmetric models and perturbative less symmetric ones.

The resulting evolution equations are of a new type compared to other models in loop quantum gravity. Although not studied in detail here, they are treatable at least by numerical schemes and provide an interesting setting to understand evolution equations in general and numerical techniques to solve them. New schemes for an efficient solution still need to be developed, also taking into account the issue of pre-classicality [34] ignored here. This condition requires solutions not to vary strongly on the scale of discreteness. It can be expected for semiclassical behaviour, but is also necessary to ensure the correct behaviour of wave packets. Techniques to extract pre-classical solutions, such as those based on generating functions [35], continued fractions [36] or numerical ones using suitable function bases [37], have been introduced but are not yet available for the equations considered here.

Also the equations themselves can be generalized in several directions. Within the same degree of symmetry one can discuss different matter choices and, at the level of the ansatz (61), more general classes for the functional behaviour of the wavefunction depending on anisotropy. Moreover, different orderings of the constraint operator, most importantly the symmetric one, can be tried. The bigger step consists in including inhomogeneous degrees of freedom perturbatively for which the analysis in this paper serves as preparation. Classically, the split into symmetric and non-symmetric variables would be given by a mode decomposition with respect to the symmetric background. A priori, there are no insurmountable difficulties in repeating the analysis here for this situation, even though technically it would be much more involved. Inhomogeneities can already be included explicitly by using midi-superspace models for which the loop framework has been constructed in [18, 38]. These situations already allow the inclusion of inhomogeneities of cosmological interest. 
Our analysis also shows that the reduced constraint of the more symmetric model still plays a role for the less symmetric one and is only amended by perturbative correction terms. Those terms are derived by expanding the less symmetric constraint operator, and not by quantizing the classically expanded constraint in the fashion of [39]. Indeed, the additional terms are much more contrived than expected classically. Although more complicated, these terms are derived from the less symmetric model and are thus well motivated. After a detailed study of the evolution, such as restrictions from quantum stability, one can draw conclusions for the form of the less symmetric constraint operator. (Such restrictions indeed arise easily as the closed isotropic model, [10] compared to [40], and the more detailed analysis in [25] showed.) Eventually, with a better understanding of the relation between models and the full theory also at the level of the Hamiltonian constraint, one can then hope to restrict possible full constraint operators and reduce their ambiguities.

\section{Acknowledgments}

We thank Jonathan Engle for discussions prior to publication of [22]. MB is grateful to the Isaac Newton Institute for Mathematical Sciences, Cambridge for hospitality during the workshop 'Global Problems in Mathematical Relativity', where this paper was completed, and thanks the organizers Piotr Chrusciel and Helmut Friedrich for an invitation. HH was supported by the fellowship A/04/21572 of Deutscher Akademischer Austauschdienst (DAAD). HAMT and HH acknowledge partial support from Mexico's National Council of Science and Technology (CONACyT), under grants CONACyT-40745-F, CONACyT-NSF-E-120-0837.

\section{Appendix A. Approximate action of operators on peaked states}

For the perturbative expansion of the Hamiltonian constraint at the quantum level, it is necessary to define and discuss an appropriate notion of expansions of operators. We are not interested in expansions valid for any state of the Hilbert space but only in expansions that can be used for states corresponding to small anisotropy such as those used in (61). Thus, the expansion parameter is not contained directly in the original operator but introduced through a restricted class of states considered for the action. We then start by choosing a space $\mathcal{D}_{\delta}$ of states in the original Hilbert space $\mathcal{H}$, or even a subset of a cylindrical space $\mathrm{Cyl}_{\mathrm{S}}$, depending on the intended perturbation parameter $\delta$ (or several such as $\varepsilon_{0}$ and $p_{\varepsilon}^{0}$ in the main part of this paper), and define

Definition 1. An operator $\hat{O}_{k}$ on $\mathcal{H}$ is an approximation of order $k$ to an operator $\hat{O}$ if $\hat{O}_{k} \Psi$ agrees, with respect to the inner product, with $\hat{O} \Psi$ for all $\Psi \in \mathcal{D}_{\delta}$ up to order $\delta^{k+1}$, i.e.

$$
\left\langle\Psi^{\prime} \mid\left(\hat{O}-\hat{O}_{k}\right) \Psi\right\rangle=O\left(\delta^{k+1}\right)
$$

for all $\Psi^{\prime} \in \mathrm{Cyl}_{\mathrm{S}}$. Agreement with respect to the inner product in this sense will be denoted by $\hat{O}_{k} \Psi \sim \hat{O} \Psi$.

To show that this is realized for the expansions performed for anisotropies in this paper, we choose $\mathcal{D}_{q_{0}, p^{0}}$ to be the span of states of the form $\Psi(q)=\sigma^{-1 / 2} s(q) \exp \left(-\left(q-q_{0}\right)^{2} / 4 \sigma^{2}\right)$ $\exp \left(\mathrm{i} p^{0} q\right.$ ) for a given $\sigma<q_{0}$ and a polynomial $s$ (we do not normalize states but keep track of factors of $\sigma$ ). Expansions performed here always lead to approximations $\hat{O}_{k}$ which are polynomials in the basic operators $\hat{q}$ and $\hat{p}$ of the anisotropy. This implies that any $\hat{O}_{k}$ considered here maps the space $\mathcal{D}_{\delta}$ to itself and can be used to show that the validity of expansions is preserved by taking products: 
Lemma 1. Let $\hat{O}_{k}^{(1)}$ and $\hat{O}_{k}^{(2)}$ be approximations of $\hat{O}^{(1)}$ and $\hat{O}^{(2)}$, respectively, such that they fix the spaces $\mathcal{D}_{\delta}$ and $\mathrm{Cyl}_{\mathrm{S}}$. Then $\hat{O}_{k}^{(1)} \hat{O}_{k}^{(2)}$ is an approximation of $\hat{O}^{(1)} \hat{O}^{(2)}$.

Proof. By assumption we have $\hat{O}^{(1)} \hat{O}^{(2)} \Psi \sim \hat{O}^{(1)} \hat{O}_{k}^{(2)} \Psi$ because we can simply replace $\Psi^{\prime}$ by $\hat{O}^{(1) \dagger} \Psi^{\prime}$ in the definition of agreement. Since $\hat{O}_{k}^{(2)} \Psi$ is in $\mathcal{D}_{\delta}$ for $\Psi \in \mathcal{D}_{\delta}$, we have

$$
\hat{O}^{(1)} \hat{O}^{(2)} \Psi \sim \hat{O}_{k}^{(1)} \hat{O}_{k}^{(2)} \Psi
$$

which finishes the proof.

We can therefore split the expansion into different steps as done in the main calculations here. This also simplifies the proof that the performed expansion of the Hamiltonian constraint is an approximation because we only need to show that multiplication operators can be approximated by simply expanding the multiplying function, which we would apply for our calculations in both the coordinate and momentum representation. We then only need

Lemma 2. Let $\hat{O} \Psi(q)=O(q) \Psi(q)$ be a multiplication operator on $\hat{H}=L^{2}(\mathbb{R}, \mathrm{d} q)$. Any partial Taylor sum $O_{k}(q)=\sum_{n=0}^{k} \frac{1}{n !} O^{(n)}(0) q^{n}$ gives rise to an approximation $\hat{O}_{k} \Psi(q)=$ $O_{k}(q) \Psi(q)$ of $\hat{O}$ order $k$ with respect to $\mathcal{D}_{\delta}$ as above.

Proof. Let $\Psi(q)=\sigma^{-1 / 2} s(q) \exp \left(-\left(q-q_{0}\right)^{2} / 4 \sigma^{2}\right)$ and $\Psi^{\prime} \in \mathrm{Cyl}_{S}$. We have

$$
\begin{aligned}
\left|\left\langle\Psi^{\prime} \mid\left(\hat{O}-\hat{O}_{k}\right) \Psi\right\rangle\right|= & \sigma^{-1 / 2}\left|\int \mathrm{d} q \bar{\Psi}^{\prime}(q)\left(O(q)-O_{k}(q)\right) s(q) \exp \left(-\left(q-q_{0}\right)^{2} / 4 \sigma^{2}\right)\right| \\
\leqslant & 2 C_{1} \sigma^{-1 / 2} \int_{q_{0}}^{q_{0}+1} \exp \left(-\left(q-q_{0}\right)^{2} / 4 \sigma^{2}\right) q^{k+1} \mathrm{~d} q \\
& +2 C_{2} \sigma^{-1 / 2} \int_{q_{0}+1}^{\infty} \exp \left(-\left(q-q_{0}\right)^{2} / 4 \sigma^{2}\right) q^{k+1} \mathrm{~d} q
\end{aligned}
$$

with positive constants $C_{1}$ and $C_{2}$ depending only on the supremum of the real and imaginary parts of $\bar{\Psi}^{\prime}(q) s(q)$. The first integral can be estimated by

$$
\begin{aligned}
\int_{q_{0}}^{q_{0}+1} \exp \left(-\left(q-q_{0}\right)^{2} / 4 \sigma^{2}\right) q^{k+1} \mathrm{~d} q & =\int_{0}^{1} \exp \left(-q^{2} / 4 \sigma^{2}\right)\left(q+q_{0}\right)^{k+1} \mathrm{~d} q \\
& =\sigma^{k+2} \int_{0}^{\sigma^{-1}} \exp \left(-q^{2} / 4\right)\left(q+q_{0} / \sigma\right)^{k+1} \mathrm{~d} q \\
& \leqslant \sigma^{k+2} \int_{0}^{\infty} \exp \left(-q^{2} / 4\right)\left(q+q_{0} / \sigma\right)^{k+1} \mathrm{~d} q
\end{aligned}
$$

where the last integral exists and does not depend strongly on $q_{0}$ and $\sigma$ since they are assumed to be of the same order. The whole expression is thus of order $\sigma^{k+2}$. For the second integral, we have

$$
\begin{aligned}
\int_{q_{0}+1}^{\infty} \exp \left(-\left(q-q_{0}\right)^{2} / 4 \sigma^{2}\right) q^{k+1} \mathrm{~d} q & =\mathrm{e}^{-(2 \sigma)^{-2}} \int_{0}^{\infty} \exp \left(-\left(q^{2}+2 q\right) / 4 \sigma^{2}\right)\left(q+q_{0}+1\right)^{k+1} \mathrm{~d} q \\
& \leqslant \mathrm{e}^{-(2 \sigma)^{-2}} \int_{0}^{\infty} \exp \left(-q^{2} / 4 \sigma^{2}\right)(q+2)^{k+1} \mathrm{~d} q
\end{aligned}
$$

where the last integral is a polynomial in $\sigma$ and the whole expression is thus of the order $\mathrm{e}^{-(2 \sigma)^{-2}}$ times a polynomial in $\sigma$. By our assumptions on the states in $\mathcal{D}_{\sigma}$, we have $\sigma$ of the order or smaller than $q_{0}$ which concludes the proof. 


\section{Appendix B. Jordan canonical form}

\section{B.1. Generalized eigenvectors and transition matrix}

In this section, we briefly review the Jordan canonical form of a square complex matrix. For further details and the proofs of the theorems, see for example [41]. We begin by stating the following

Theorem 1. If a square matrix A of order $n$ has s linearly independent eigenvectors, then it is similar to a matrix $J$ of the following form, called a Jordan canonical form

$$
J=S^{-1} A S=\left(\begin{array}{cccc}
J_{1} & & & 0 \\
& J_{2} & & \\
& & \ddots & \\
0 & & & J_{S}
\end{array}\right),
$$

in which each $J_{i}$, called the Jordan block, is a triangular matrix of the form

$$
J_{i}=\left(\begin{array}{cccc}
\lambda_{i} & 1 & & 0 \\
& \ddots & \ddots & \\
& & \ddots & 1 \\
0 & & & \lambda_{i}
\end{array}\right),
$$

where $\lambda_{i}$ is a single eigenvalue of A. Each Jordan block corresponds to a linearly independent eigenvector.

This theorem states that we can bring any square matrix to its Jordan form. The usefulness of this form lies in the fact that it is easier to obtain powers of the matrix $A$, as can be seen by taking a look at the form of $J$ in (B.1) and (B.2). The matrix $S$ in (B.1) is called the transition matrix. Now we state the procedure to obtain both matrices $J$ and $S$.

The basic elements to obtain the Jordan form are the generalized eigenvectors, replacing ordinary eigenvectors of a diagonalizable matrix.

Definition 2. A nonzero vector $\mathbf{x}$ is said to be a generalized eigenvector of $A$ of rank $k$ belonging to an eigenvalue $\lambda$ if

$$
(A-\lambda I)^{k} \mathbf{x}=\mathbf{0} \quad \text { and } \quad(A-\lambda I)^{k-1} \mathbf{x} \neq \mathbf{0} .
$$

If $k=1$ this is the usual definition of an eigenvector. For a generalized eigenvector $\mathbf{x}$ of rank larger than 1 belonging to the eigenvalue $\lambda$ define

$$
\begin{aligned}
& \mathbf{x}_{k}=\mathbf{x}, \\
& \mathbf{x}_{k-1}=(A-\lambda I) \mathbf{x}=(A-\lambda I) \mathbf{x}_{k}, \\
& \mathbf{x}_{k-2}=(A-\lambda I)^{2} \mathbf{x}=(A-\lambda I) \mathbf{x}_{k-1}, \\
& \vdots \\
& \mathbf{x}_{2}=(A-\lambda I)^{k-2} \mathbf{x}=(A-\lambda I) \mathbf{x}_{3}, \\
& \mathbf{x}_{1}=(A-\lambda I)^{k-1} \mathbf{x}=(A-\lambda I) \mathbf{x}_{2} .
\end{aligned}
$$

One can check that each one of the $\mathbf{x}_{j}$ above is a generalized eigenvector of the eigenvalue $\lambda$. 
Definition 3. The set of vectors $\left\{\mathbf{x}_{1}, \ldots, \mathbf{x}_{k}\right\}$ is called a chain of generalized eigenvectors belonging to the eigenvalue $\lambda$.

Now the transition matrix $S$ can be constructed from the chains of linearly independent generalized eigenvectors of $A$ [41].

\section{B.2. Powers of square matrices}

The Jordan canonical form of any square matrix $A$ enables us to compute its powers $A^{k}$. Let $J$ be the Jordan canonical form of an arbitrary $n \times n$ matrix $A$ such that

$$
S^{-1} A S=J=\left(\begin{array}{ccc}
J_{1} & & \\
& \ddots & \\
& & J_{S}
\end{array}\right),
$$

where $S$ is made of the generalized eigenvectors of $A$ and $J_{S}$ are Jordan blocks.

Since we have

$$
A^{k}=S^{-1} J^{k} S=S\left(\begin{array}{ccc}
J_{1}^{k} & & \\
& \ddots & \\
& & J_{s}^{k}
\end{array}\right) S^{-1}
$$

for $k=1,2, \ldots$, it is enough to compute $J^{k}$ for each Jordan block $J$. An $m \times m$ Jordan block $J$ belonging to an eigenvalue $\lambda$ of $A$ may be written as

$$
J=\left(\begin{array}{cccc}
\lambda & 1 & & 0 \\
0 & \ddots & \ddots & \\
& & \lambda & 1 \\
0 & & 0 & \lambda
\end{array}\right)=\lambda \mathbb{I}_{m \times m}+\left(\begin{array}{cccc}
0 & 1 & \cdots & 0 \\
0 & \ddots & \ddots & 0 \\
\vdots & & 0 & 1 \\
0 & \cdots & 0 & 0
\end{array}\right)=\lambda \mathbb{I}+N,
$$

where $\mathbb{I}$ is the identity matrix and $N$ is nilpotent. Clearly we have

$$
J^{k}=(\lambda \mathbb{I}+N)^{k}=\sum_{j=0}^{k}\left(\begin{array}{l}
k \\
j
\end{array}\right) \lambda^{k-j} N^{j} .
$$

But $N^{k}=0$ for $k \geqslant m$ and thus, by defining $\left(\begin{array}{l}k \\ l\end{array}\right)=0$ if $k<l$

$J^{k}=\sum_{j=0}^{m-1}\left(\begin{array}{l}k \\ j\end{array}\right) \lambda^{k-j} N^{j}=\lambda^{k} \mathbb{I}+k \lambda^{k-1} N+\cdots+\left(\begin{array}{c}k \\ m-1\end{array}\right) \lambda^{k-(m-1)} N^{m-1}$.

\section{References}

[1] DeWitt B S 1967 Quantum theory of gravity: I. The canonical theory Phys. Rev. $1601113-48$

[2] Wiltshire D L 1996 An introduction to quantum cosmology Cosmology: The Physics of the Universe ed B Robson, N Visvanathan and W S Woolcock (Singapore: World Scientific)pp 473-531 (Preprint gr-qc/0101003)

[3] Bojowald M and Morales-Técotl H A 2004 Cosmological applications of loop quantum gravity Proc. 5th Mexican School (DGFM): The Early Universe and Observational Cosmology (Lect. Notes Phys. vol 646) (Berlin: Springer) pp 421-62 (Preprint gr-qc/0306008)

[4] Bojowald M 2005 Loop quantum cosmology 100 Years of Relativity-Space-Time Structure and Beyond ed A Ashtekar (Singapore: World Scientific) (Preprint gr-qc/0505057)

[5] Bojowald M 2005 Loop quantum cosmology Living Rev. Rel. 811 
[6] Bojowald M 2006 Degenerate configurations, singularities and the non-Abelian nature of loop quantum gravity Class. Quantum Grav. 23 987-1008 (Preprint gr-qc/0508118)

[7] Bojowald M 2003 Homogeneous loop quantum cosmology Class. Quantum Grav. $202595-615$ (Preprint gr-qc/0303073)

[8] Bojowald M, Date G and Vandersloot K 2004 Homogeneous loop quantum cosmology: the role of the spin connection Class. Quantum Grav. 21 1253-78 (Preprint gr-qc/0311004)

[9] Ashtekar A and Bojowald M 2006 Quantum geometry and the Schwarzschild singularity Class. Quantum Grav. 23 391-411 (Preprint gr-qc/0509075)

[10] Bojowald M 2002 Isotropic loop quantum cosmology Class. Quantum Grav. $192717-41$ (Preprint gr-qc/ 0202077)

[11] Bojowald M 2001 Absence of a singularity in loop quantum cosmology Phys. Rev. Lett. 86 5227-30 (Preprint gr-qc/0102069)

[12] Ashtekar A, Bojowald M and Lewandowski J 2003 Mathematical structure of loop quantum cosmology Adv. Theor. Math. Phys. 7 233-68 (Preprint gr-qc/0304074)

[13] Ashtekar A 1987 New Hamiltonian formulation of general relativity Phys. Rev. D $361587-602$

[14] Barbero G F J 1995 Real Ashtekar variables for Lorentzian signature space-times Phys. Rev. D 51 5507-10 (Preprint gr-qc/9410014)

[15] Immirzi G 1997 Real and complex connections for canonical gravity Class. Quantum Grav. 14 L177-81

[16] Ashtekar A, Lewandowski J, Marolf D, Mourão J and Thiemann T 1995 Quantization of diffeomorphism invariant theories of connections with local degrees of freedom J. Math. Phys. 36 6456-93 (Preprint gr-qc/9504018)

[17] Bojowald M and Kastrup H A 2000 Symmetry reduction for quantized diffeomorphism invariant theories of connections Class. Quantum Grav. 17 3009-43 (Preprint hep-th/9907042)

[18] Bojowald M 2004 Spherically symmetric quantum geometry: states and basic operators Class. Quantum Grav. 21 3733-53 (Preprint gr-qc/0407017)

[19] Bojowald M 2001 Loop quantum cosmology: III. Wheeler-DeWitt operators Class. Quantum Grav. 18 1055-70 (Preprint gr-qc/0008052)

[20] Bojowald M 2001 The semiclassical limit of loop quantum cosmology Class. Quantum Grav. 18 L109-16 (Preprint gr-qc/0105113)

[21] Ashtekar A, Fairhurst S and Willis J 2003 Quantum gravity, shadow states, and quantum mechanics Class. Quantum Grav. 20 1031-62 (Preprint gr-qc/0207106)

[22] Engle J 2006 Quantum theory and its symmetry reduction Class. Quantum Grav. 23 2861-93

[23] Thiemann T 1998 QSD: V. Quantum gravity as the natural regulator of matter quantum field theories Class. Quantum Grav. 15 1281-314 (Preprint gr-qc/9705019)

[24] Bojowald M 2001 Inverse scale factor in isotropic quantum geometry Phys. Rev. D 64084018 (Preprint gr-qc/0105067)

[25] Bojowald M and Date G 2004 Consistency conditions for fundamentally discrete theories Class. Quantum Grav. 21 121-43 (Preprint gr-qc/0307083)

[26] Hertog T and Horowitz G T 2005 Holographic description of AdS cosmologies J. High Energy Phys. JHEP04(2005)005 (Preprint hep-th/0503071)

[27] Bojowald M 2005 Non-singular black holes and degrees of freedom in quantum gravity Phys. Rev. Lett. 95061301 (Preprint gr-qc/0506128)

[28] Brunnemann J and Thiemann T 2006 On (cosmological) singularity avoidance in loop quantum gravity Class. Quantum Grav. 23 1395-427 (Preprint gr-qc/0505032)

[29] Singh P and Toporensky A 2004 Big crunch avoidance in $k=1$ semi-classical loop quantum cosmology Phys. Rev. D 69104008 (Preprint gr-qc/0312110)

Vereshchagin G V 2004 Qualitative approach to semi-classical loop quantum cosmology J. Cosmol. Astropart. Phys. JCAP07(2004)013 (Preprint gr-qc/0406108)

Bojowald M, Maartens R and Singh P 2004 Loop quantum gravity and the cyclic universe Phys. Rev. D 70083517 (Preprint hep-th/0407115)

Date G and Hossain G M 2005 Genericity of big bounce in isotropic loop quantum cosmology Phys. Rev. Lett. 94011302 (Preprint gr-qc/0407074)

Date G 2005 Absence of the Kasner singularity in the effective dynamics from loop quantum cosmology Phys. Rev. D 71127502 (Preprint gr-qc/0505002)

Ashtekar A, Pawlowski T and Singh P 2006 Quantum nature of the big bang Preprint gr-qc/0602086

[30] Lidsey J E, Mulryne D J, Nunes N J and Tavakol R 2004 Oscillatory universes in loop quantum cosmology and initial conditions for inflation Phys. Rev. D 70063521 (Preprint gr-qc/0406042)

Mulryne D J, Nunes N J, Tavakol R and Lidsey J 2005 Inflationary cosmology and oscillating universes in loop quantum cosmology Int. J. Mod. Phys. A 20 2347-57 (Preprint gr-qc/0411125) 
Mulryne D J, Tavakol R, Lidsey J E and Ellis G F R 2005 An emergent universe from a loop Phys. Rev. D 71123512 (Preprint astro-ph/0502589)

Bojowald M 2005 Original questions Nature 436 920-1

Nunes N J 2006 Inflation: a graceful entrance from loop quantum cosmology Phys. Rev. D 72103510 (Preprint astro-ph/0507683)

[31] Bojowald M 2002 Inflation from quantum geometry Phys. Rev. Lett. 89261301 (Preprint gr-qc/0206054)

Bojowald M, Singh P and Skirzewski A 2004 Coordinate time dependence in quantum gravity Phys. Rev. D 70124022 (Preprint gr-qc/0408094)

Date G and Hossain G M 2004 Effective Hamiltonian for isotropic loop quantum cosmology Class. Quantum Grav. 21 4941-53 (Preprint gr-qc/0407073)

Banerjee K and Date G 2005 Discreteness corrections to the effective Hamiltonian of isotropic loop quantum cosmology Class. Quantum Grav. 22 2017-33 (Preprint gr-qc/0501102)

Singh P and Vandersloot K 2005 Semi-classical states, effective dynamics and classical emergence in loop quantum cosmology Phys. Rev. D 72084004 (Preprint gr-qc/0507029)

Ashtekar A, Bojowald $\mathrm{M}$ and Willis $\mathrm{J}$, in preparation

Willis J 2004 On the low-energy ramifications and a mathematical extension of loop quantum gravity PhD Thesis The Pennsylvania State University

Bojowald M and Skirzewski A 2005 Effective equations of motion for quantum systems Preprint math-ph/0511043

[32] Belinskii V A, Khalatnikov I M and Lifschitz E M 1982 A general solution of the Einstein equations with a time singularity Adv. Phys. 13 639-67

[33] Bojowald M and Date G 2004 Quantum suppression of the generic chaotic behaviour close to cosmological singularities Phys. Rev. Lett. 92071302 (Preprint gr-qc/0311003)

Bojowald M, Date G and Hossain G M 2004 The Bianchi IX model in loop quantum cosmology Class. Quantum Grav. 21 3541-69 (Preprint gr-qc/0404039)

[34] Bojowald M 2001 Dynamical initial conditions in quantum cosmology Phys. Rev. Lett. 87121301 (Preprint gr-qc/0104072)

[35] Cartin D, Khanna G and Bojowald M 2004 Generating function techniques for loop quantum cosmology Class. Quantum Grav. 21 4495-509 (Preprint gr-qc/0405126)

Cartin D and Khanna G 2005 Absence of pre-classical solutions in Bianchi I loop quantum cosmology Phys. Rev. Lett. 94111302 (Preprint gr-qc/0501016)

Date G 2005 Pre-classical solutions of the vacuum Bianchi I loop quantum cosmology Phys. Rev. D 72067301 (Preprint gr-qc/0505030)

[36] Bojowald M and Rej A 2005 Asymptotic properties of difference equations for isotropic loop quantum cosmology Class. Quantum Grav. 22 3399-420 (Preprint gr-qc/0504100)

[37] Connors S and Khanna G 2005 Approximate pre-classical solutions in loop quantum cosmology Preprint gr-qc/0509081

[38] Bojowald M and Swiderski R, 2006 Spherically symmetric quantum geometry: Hamiltonian constraint Class. Quantum Grav. 23 2129-54

[39] Halliwell J J and Hawking S W 1985 Origin of structure in the universe Phys. Rev. D 31 1777-91

[40] Bojowald M and Vandersloot K 2003 Loop quantum cosmology, boundary proposals, and inflation Phys. Rev. D 67124023 (Preprint gr-qc/0303072)

[41] Zhang F (ed) 1999 Matrix Theory (UTX Series) (New York: Springer) 